NASA Contractor Report 198501

AIAA-96-0807

\title{
The Modelling of Symmetric Airfoil Vortex Generators
}

B.J. Wendt

Modern Technologies Corporation

Middleburg Heights, Ohio

and

B.A. Reichert

Kansas State University

Manhattan, Kansas

June 1996

Prepared for

Lewis Research Center

Under Contract NAS3-27377

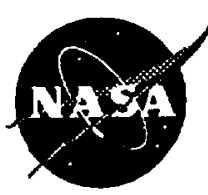

National Aeronautics and

Space Administration 


\title{
THE MODELLING OF SYMMETRIC AIRFOIL VORTEX GENERATORS
}

\author{
B. J. Wendt', \\ Modern Technologies Corporation, Middleburg Heights, Ohio, 44130 \\ B. A. Reichert ${ }^{\ddagger}$ \\ Kansas State University, Manhattan, Kansas, 66506
}

Abstract

An experimental study is conducted to determine the dependence of vortex generator geometry and impinging flow conditions on shed vortex circulation and crossplane peak vorticity for one type of vortex generator. The vortex generator is a symmetric airfoil having a NACA 0012 cross-sectional profile. The geometry and flow parameters varied include angle-of-attack $\alpha$, chordlength $c$, span $h$, and Mach number $M$.

The vortex generators are mounted either in isolation or in a symmetric counter-rotating array configuration on the inside surface of a straight pipe. The turbulent boundary layer thickness to pipe radius ratio is $\delta / R \approx$ 0.17 . Circulation and peak vorticity data are derived from crossplane velocity measurements conducted at or about 1 chord downstream of the vortex generator trailing edge.

Shed vortex circulation is observed to be proportional to $M, \alpha$, and $h / \delta$. With these parameters held constant, circulation is observed to fall off in monotonic fashion with increasing airfoil aspect ratio $A R$. Shed vortex peak vorticity is also observed to be proportional to $M, \alpha$, and $h / \delta$. Unlike circulation, however, peak vorticity is observed to increase with increasing aspect ratio, reaching a peak value at $A R \approx 2.0$ before falling off.

\section{Introduction}

Vortex generators are often used in a variety of fluid engineering applications where a small, but critical, amount of flow control is necessary for proper flow component performance.Common uses are in aircraft systems where surface-mounted vortex generators are applied in external (airframe related) and internal (propulsion related) flows. Figure 1 depicts a typical application of surface-mounted vortex generators. In external flow situations, such as that encountered on the wing surface in Figure 1, the most common arrangement is array formations upstream of flight control surfaces where boundary layer attachment is often critical to flight performance. An array of vortex generators is also being used in the

\footnotetext{
"Research Engineer, 7530 Lucerne Drive, Islander Two, Suite 206, Member AIAA

${ }^{\ddagger}$ Associate Professor, Department of Mechanical Engineering, 302 Durland Hall, Member AIAA
}

aircraft inlet in Figure 1. In internal flows, vortex generators are used to prevent excessive boundary layer growth, flow separation, and to reduce total pressure distortion of the airstream ingested by the aircraft engine. These effects occur readily in inlet ducts and diffusers due to such factors as duct centerline curvature, and large streamwise variations in duct cross-sectional area.

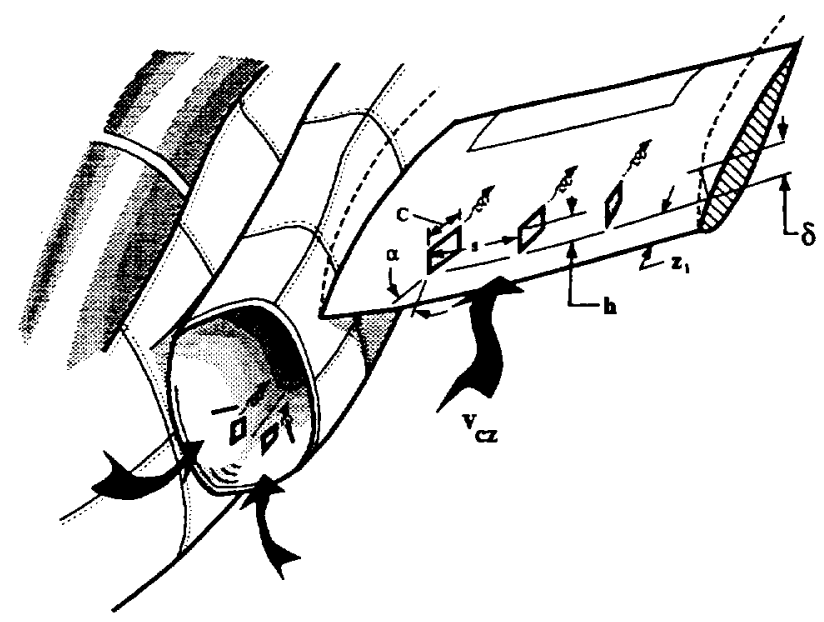

Figure 1 Flow conditioning with vortex generators.

The key to vortex generator performance is in the mixing and secondary velocity field created downstream by the shed vortex structures. If properly situated in the flowfield, the helical motion of the fluid in the vortex forces high energy fluid of the freestream or core flow into the slower moving fluid of the boundary layer. The re-energized boundary layer fluid is now more resistant to flow separation. Vortex generator induced flow may also be used to counter boundary layer thickening due to component-generated secondary flows. Recent experimental work in an S-duct ${ }^{1}$ and a rectangular-tosemiannular diffuser ${ }^{2}$ have demonstrated the effectiveness of this approach.

The large number of parameters to consider when designing a vortex generator array for an aircraft component (such as the wing or inlet illustrated in Figure 1) has the implication that experimental work to achieve optimum performance is often slow and expensive. This fact has motivated a few workers in computational fluid mechanics to assist in the optimizing process by including a means of representing vortex generators in their codes. A simple and effective way of doing this is to employ a model for the crossplane velocity or vorticity field induced by the vortex generators. This is the approach taken in recent work by Anderson et al..$^{3.4}$ who examined multiple vortex generator array geometries in a 
diffusing S-duct inlet using a parabolized Navier-Stokes (RNS) solver. A similar inclusion of embedded vortices in a full Navier-Stokes (FNS) code was implemented by Cho and Greber ${ }^{5}$ for a constant area circular duct and a diffusing S-duct geometry.

The advantage to modelling the crossplane velocity or vorticity distribution induced by the vortex generator is that a simple exponential expression accurately captures the crossplane velocity or vorticity distribution of the shed vortex. To demonstrate this, consider Figure 2. Figure 2 illustrates the secondary velocity field of an embedded vortex (data on the left, model on the right) shed from an airfoil-type vortex generator. This vortex generator is one of 12 symmetrically placed vortex generators spanning the inside circumference of a straight pipe. The data is acquired in the crossplane one chord length downstream of the vortex generators. The model is constructed from a summation of 24 terms (12 embedded vortices plus 12 image vortices) each having the form ${ }^{6}$ :

$$
\Gamma_{i}\left[1-\exp \left(\frac{-\pi \omega_{i}^{\max }}{\Gamma_{i}}\right) R_{i}^{2}\right],
$$

where $\Gamma_{i}$ is the measured circulation of the $i$ th vortex or image, $\omega_{i}^{\text {max }}$ is the measured peak or maximum value of the induced streamwise vorticity field, and $R_{i}$ is the measured crossplane location of the vortex center. These quantities are known as "vortex descriptors" following the work of Russell Westphal' ${ }^{7}$. Any such model requires a description of the vortex strength (here $\Gamma_{i}$ ) and concentration (here $\omega_{i}^{\max }$, but viscous core radius, $r_{c i}$, is another possibility). The model above is based on the classical "ideal viscous" or Oseen vortex. ${ }^{8}$

Many studies have demonstrated the remarkable similarities between the Oseen-based model and the crossplane structure of embedded vortices shed from vortex generators. Pauley and Eaton ${ }^{9}$ demonstrated this in a study of delta wing vortex generators, Wendt et al. ${ }^{10}$ in a study of blade-type vortex generators, Wendt and Hingst ${ }^{8}$ for low-profile wishbone vortex generators, and more recently, Wendt et al. ${ }^{6}$ on symmetric airfoil-type vortex generators.

To complete the modelling of a vortex generator we must now show how the descriptors of the model vortex depend on downstream axial position, the geometry of the vortex generator, and impinging flow conditions. For airfoil-type vortex generators we might express this dependence as:

$$
\begin{aligned}
\Gamma & =\Gamma(z, c, h, \alpha, \text { profile, } \delta, M, R e, \ldots), \\
\omega^{\max } & =\omega^{\max }(z, c, h, \alpha, \text { profile, } \delta, M, R e, \ldots), \\
R & =R(z, c, h, \alpha, \text { profile, } \delta, M, R e, \ldots),
\end{aligned}
$$

where "profile" refers to the shape of the airfoil, $z$ is the axial position (measured from the trailing edge of the vortex generator), $c$ is the chord length, $h$ is the span, $\alpha$ is the angle-of-attack, $\delta$ is the boundary layer thickness of the impinging stream, $M$ is the freestream or core Mach number and $R e$ is a Reynolds number. The vortex generator models in use at the present time only crudely approximate the functional dependence indicated in Equation 2. As a means of improving current vortex generator models this study was undertaken to examine the effect of vortex generator geometry and impinging flow conditions for one type of symmetric airfoil vortex generator (NACA 0012). This paper reports the results of a series of experimental tests covering a range of vortex generator $h / c$ (or "aspect ratio"), $h / \delta, \alpha$, and $M$. The vortex generators are mounted either in isolation on the inside surface of a straight pipe, or in a symmetric counter-rotating array spanning the full circumference of the pipe. Duct or pipe core flow conditions are subsonic with a nominal boundary layer thickness to pipe diameter ratio, $\delta / D=0.09$. Three-dimensional mean flow velocity data are acquired in a crossplane grid located approximately one chordlength downstream of the vortex generator model(s). This is done using a rake of

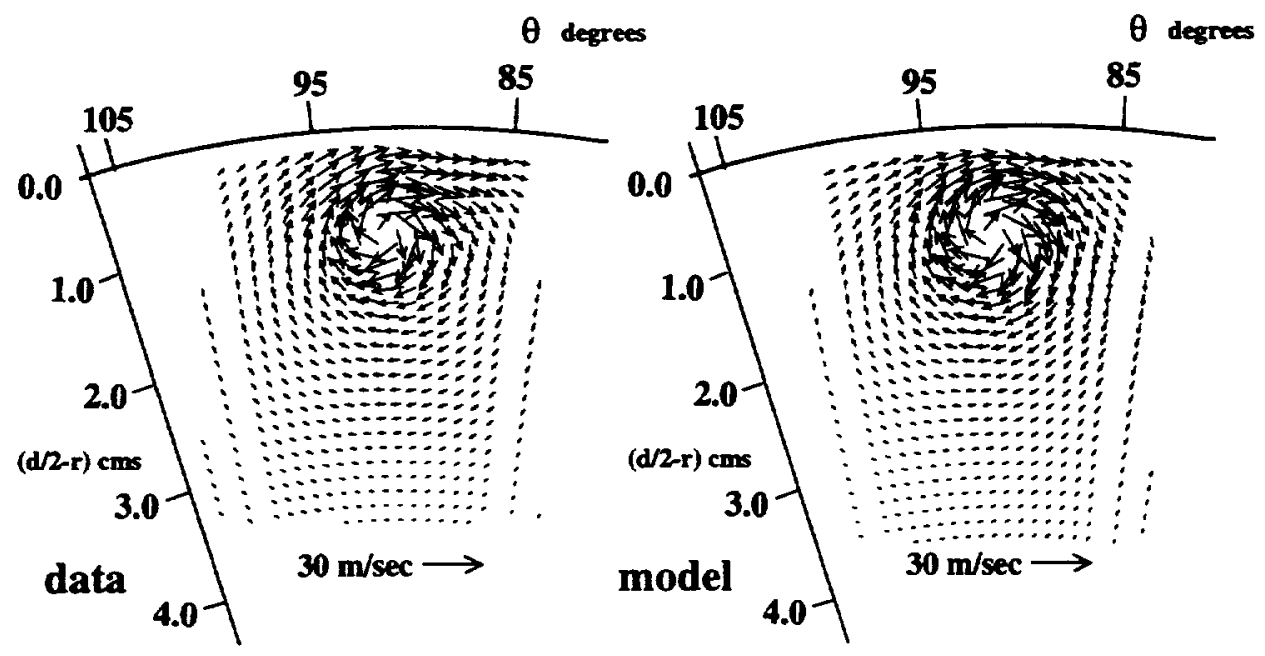

Figure 2 A comparison of the transverse velocity field of an embedded vortex; data on the left, and a model constructed from the superposition of Oseen vortices on the right. 


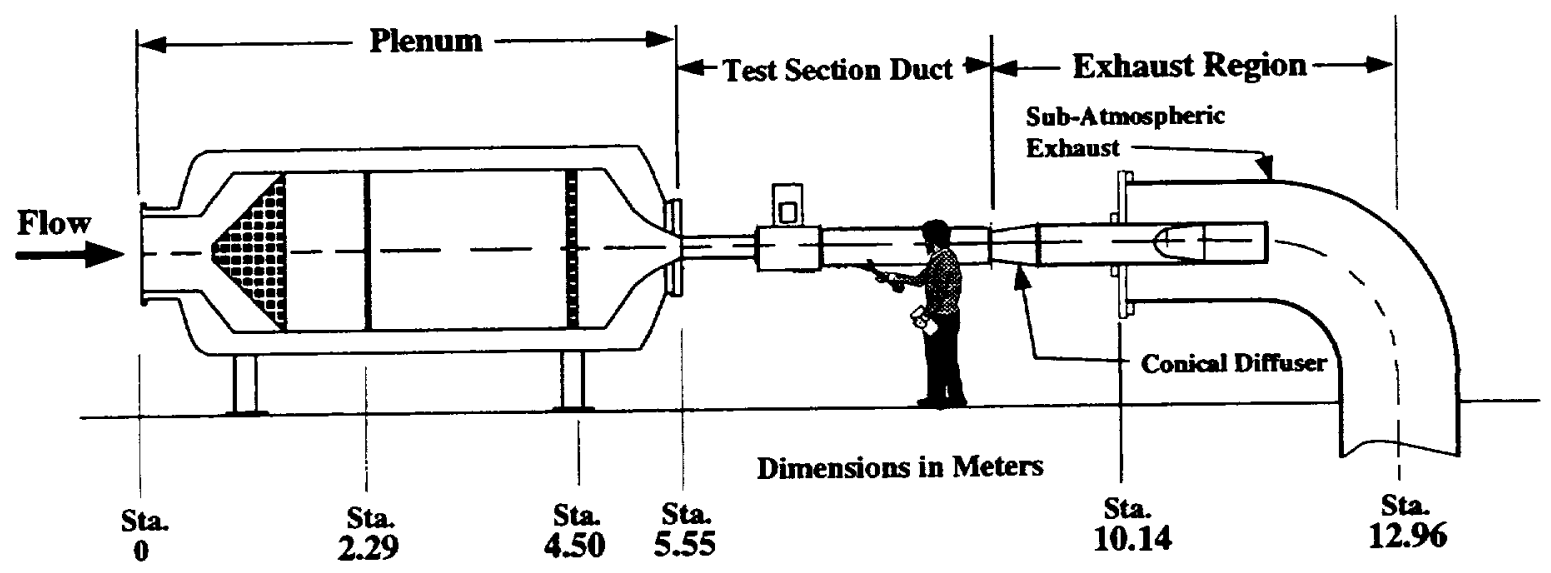

Figure 3 The Internal Fluid Mechanics Facility of NASA Lewis.

seven-hole probes. A total of 14 test cases are considered. Comparisons of the experimental results with expressions derived from inviscid airfoil theory will be conducted.

The influence of axial $(z)$ decay was considered in a previous report, Reference 6 . In Reference 6 the vortices were generated in a counter-rotating array configuration and axial decay was examined to a position of 17.25 chordlengths downstream of the array trailing edge. In addition to the parametric study described above, the axial decay of a single embedded vortex is examined here for one condition of vortex generator geometry and flow variable. These results are compared to the axial decay results presented in Reference 6.

\section{Facilities and Procedures}

Test Facility

This study was conducted in the Internal Fluid Mechanics Facility (IFMF) of NASA Lewis. The IFMF is a subsonic facility designed to investigate a variety of duct flow phenomena. The facility, as it is configured for this test, is illustrated in Figure 3. Air is supplied from the surrounding test cell to a large settling chamber containing a honeycomb flow straightener and screens. At the downstream end of the settling chamber the airstream is accelerated through a contraction section (having a crosssectional area reduction of 59 to 1) to the test section duct. The test section duct consists of a straight circular pipe of inside diameter $d=20.4 \mathrm{cms}$. After exiting the test section duct, the airstream enters a short conical diffuser and is then routed to a discharge plenum which is continuously evacuated by central exhauster facilities. The Mach number range in the test section duct is between 0.2 and 0.8 with corresponding Reynolds numbers (based on pipe diameter) between 0.95 and 3.80 million. Mass flows are between 3 and $7 \mathrm{kgs} / \mathrm{sec}$. More information on the design and operation of the IFMF may be found in the report of Porro et al. ${ }^{11}$

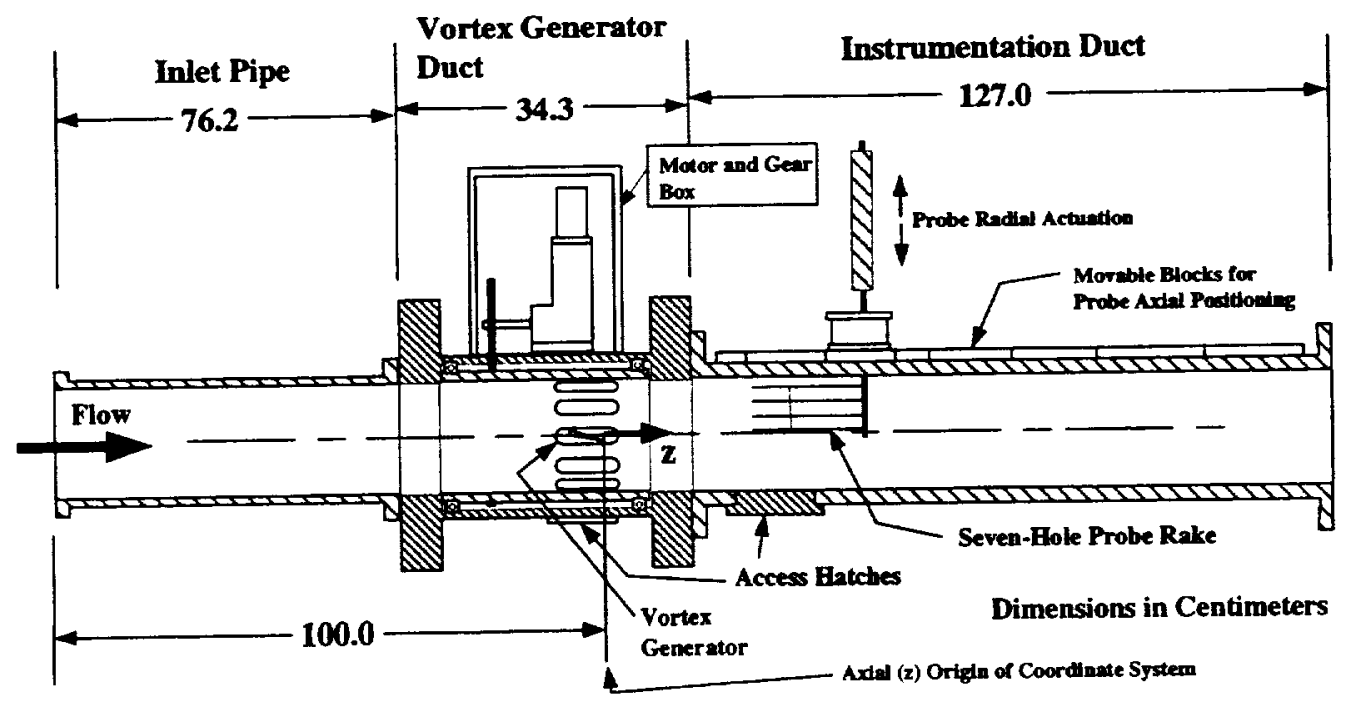

Figure 4 A cut-away sketch of the test section duct. 
Research Instrumentation and Test Parameters

Figure 4 is a detailed sketch of the various test section components.

A short section of straight pipe (labelled "inlet pipe" in Figure 4) connects the exit of the facility contraction to the duct segment containing the vortex generator model(s). Static pressure taps located on the surface of the inlet pipe allow the nominal core Mach number in the test section to be set and monitored.

The duct portion with the vortex generator(s) is referred to as the "vortex generator duct". The inside surface of the vortex generator duct (and hence the attached vortex generator) rotates about an axis coinciding with the test section centerline. 360 degrees of rotation is possible. The rotation is driven by a motor and gear located in an airtight box above the vortex generator duct.

A typical vortex generator is illustrated in Figure 5. These vortex generators consist of an airfoil-shaped blade (with a NACA 0012 profile) mounted perpendicular to the surface of a base plug. The surface of the base plug is contoured to the inside radius of the vortex generator duct. Both blade and plug are machined from an aluminum alloy using a wire cutting (electric discharge machining) process. Figure 5 includes a table listing the chord, span, and angle-of-attack of the vortex generator models examined in this study. The baseline or "reference" model has a chordlength $c_{0}=4.06 \mathrm{cms}$, a span $h_{0}=1.02$ $\mathrm{cms}$, and an angle-of-attack, $\alpha_{0}=16^{\circ}$.

To study variation in vortex generator span, chord, and angle-of-attack, the vortex generator models are mounted in isolation. To study variation in core Mach number it is necessary to test the vortex generators in symmetric counter-rotating array configurations. This

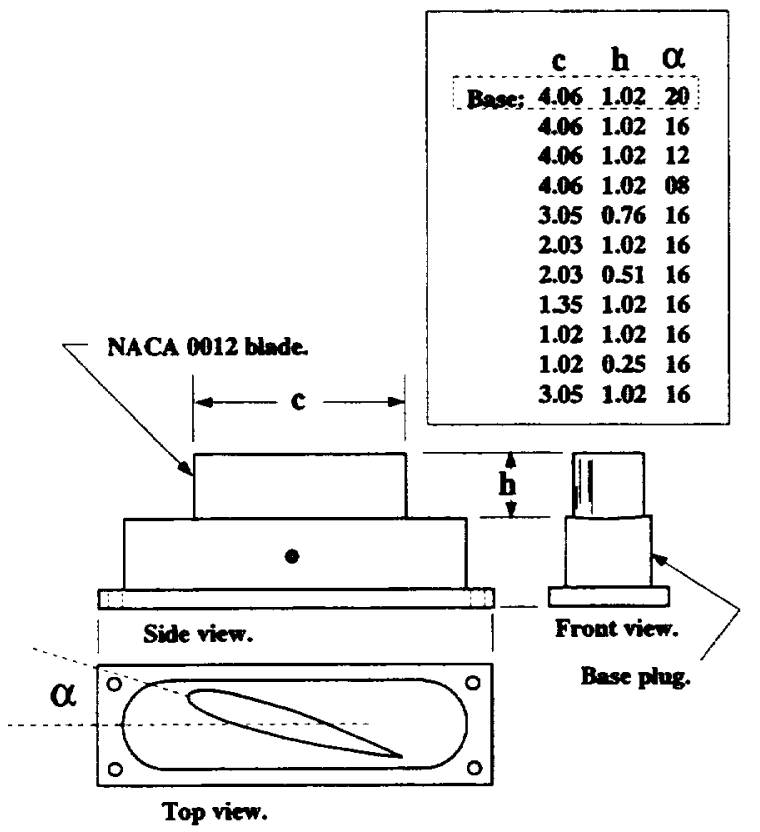

Figure 5 The airfoil vortex generator. Dimensions are in centimeters, and angles are in degrees. is due to a torque-balancing problem at Mach numbers above 0.4 ; i.e. the lift force-generated torque produced by an airfoil mounted in isolation overwhelms the restraining torque provided by the turning gear and motor. Figure 6 illustrates the array configuration used to study variation in core Mach number. The vortex generator array consists of 12 blades, identical in geometry ( $h=1.02$ $\mathrm{cms}, c=4.06 \mathrm{cms}$ ), but alternating in angle-of-attack, $\alpha= \pm 16^{\circ}$. As seen in the downstream view of Figure 6 , the vortex generators are equally spaced, circumferentially, at mid-chord. $\Delta \theta_{b}$ between the mid-chord position of adjacent blades is 30 degrees, where $\theta$ is the circumferential coordinate.

The coordinate system used in this study originates in the vortex generator duct. $z=0$ coincides with the trailing edge of the vortex generator or vortex generator array. The axial location of downstream $(r, \theta)$ crossplanes are given in terms of blade chord, $c$. For example, the first survey location possible (with the reference vortex generator installed) is the $(r, \theta)$ crossplane located at $z=0.38 c_{0}$.

The duct segment downstream of the vortex generator duct is stationary (non-rotating). This test section segment is referred to as the "instrumentation duct". The flowfield measurements are acquired in this duct through the use of a radially actuated rake-probe indicated in Figure 4. To acquire data in an $(r, \theta)$ crossplane, the rakeprobe is actuated over a radial segment extending from the duct wall to the duct centerline. The vortex generator duct and vortex generator array are then rotated an increment in circumferential position, $\Delta \theta$, and the radial survey repeated. In this manner pie shaped pieces of the flowfield are examined. A narrow slot running the approximate length of the instrumentation duct allows the rake-probe to be located at various downstream cross-

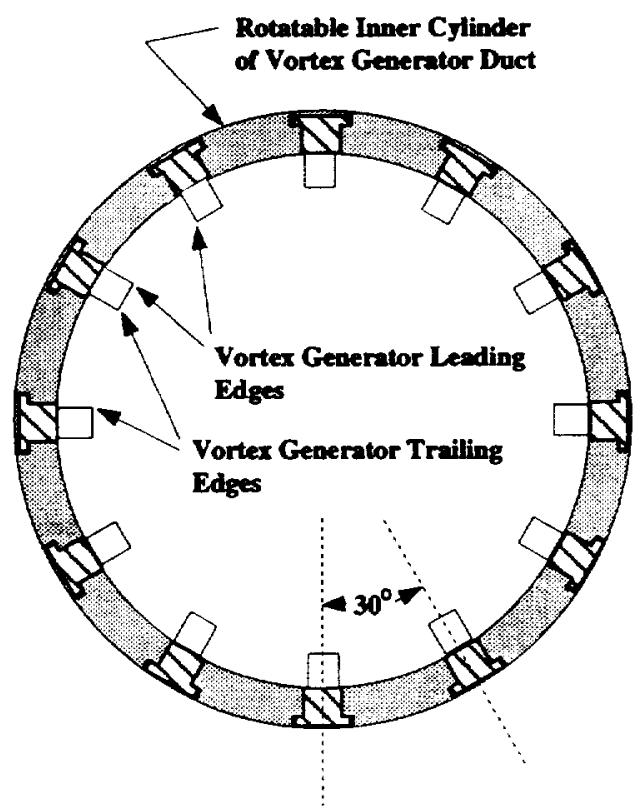

Figure 6 The vortex generator array. 
planes. A series of slot-sealing blocks determines the allowable axial location of survey crossplanes, $z_{i}$ (again with the reference vortex generator mounted) :

$$
\begin{gathered}
z_{i}=0.38 c_{0}, 1.00 c_{0}, \ldots, 10.38 c_{0} \\
14.75 c_{0}, 15.38 c_{0}, \ldots, 24.75 c_{0}
\end{gathered}
$$

The rake-probe consists of 4 seven-hole probe tips spaced $2.54 \mathrm{cms}$ apart. These probes are calibrated in accordance with the procedure outlined by Zilliac. ${ }^{12}$ The flow angle range covered in calibration is $\pm 60^{\circ}$ in both pitch and yaw for the probe tip closest to the wall. The calibration range for the outer 3 tips is approximately $\pm 30^{\circ}$. Uncertainty in flow angle measurement is $\pm 0.7^{\circ}$ in either pitch or yaw, for flow angle magnitude below 35 degrees (pitch and yaw flow angle magnitude did not exceed 35 degrees in this study). The corresponding uncertainty in velocity magnitude is approximately $\pm 1 \%$ of the core velocity, $v_{z c}$.

The circumferential extent of the crossplane survey grid is determined by the objectives of the study. In Reference 6 we were interested in studying the crossplane domain of a single embedded vortex constrained on either side by its counter-rotating neighbors. The circumferential extent of that domain was 30 degrees as determined by the geometry of the parent vortex generator array. Surveying the full 30 degrees allowed us to determine quantities such as vortex angular momentum and transverse kinetic energy, in addition to the vortex core descriptors of circulation and peak vorticity. This data will again be useful at a later point in this paper when we attempt an analysis of the present results. We note now, however, that acquiring vortex angular momentum or transverse kinetic energy for an isolated embedded vortex would involve surveying most, if not all, of the pipe crossplane, an impractical requirement considering the crossplane grid resolution employed here. Thus, for most of the present study, we will limit ourselves to obtaining only the vortex descriptors. These can be accurately measured by a survey grid covering the near-region of the vortex viscous core. In most instances this involves a grid of only 20 or 25 degrees in circumferential extent.

Crossplane grid resolution is based both on the size of the vortex core and the time needed to acquire data with the rake-probe. In most test cases here the axial location of the survey grid is $z=c_{0}$, the vortex core is approximately 1 centimeter in diameter, and is highly concentrated with large secondary velocities present. Sufficient resolution is obtained with $\Delta \theta=1^{\circ}$ on the grid interior, and $\Delta r=1.3 \mathrm{~mm}$. The vortex core grows in size and becomes more diffuse as it moves away from the parent vortex generator. Thus for axial locations sufficiently far downstream (visited when examining the axial decay of the single embedded vortex) we can coarsen the grid described above somewhat, with $\Delta \theta=1.5^{\circ}$ and $\Delta r=1.7 \mathrm{~mm}$.

\section{Experimental Results}

Table 1 and Figures $7-11$ summarize the results of this study. Table 1 lists the test conditions for every crossplane illustrated in Figures $7-11$, and tabulates the vortex descriptors determined for each crossplane of data. The vortex descriptors are also included in Figures 7-11. In Figures $7-11$ the radial axis represents distance from the wall, in centimeters, and the circumferential axis represents angular position in degrees. The axial position of each crossplane is indicated. Note again that this is given in chordlengths of the model being tested and is measured from the trailing edge tip of the airfoil.

The vortex descriptors originate from the transverse velocity data in the crossplane. The vector plots on the left hand side of Figures $7-11$ are the measured transverse velocity data. The transverse velocity data is first converted to streamwise vorticity data following the relation:

$$
\omega_{z}=\frac{\delta v_{\theta}}{\delta r}+\frac{v_{\theta}}{r}-\frac{1}{r} \frac{\delta v_{r}}{\delta \theta}
$$

where $\left(v_{r}, v_{\theta}\right)$ are the transverse components of velocity in the radial and circumferential coordinates, respectively. Finite difference formulas are used to represent the spatial derivatives in Equation 4. The resulting streamwise vorticity fields are plotted on the right hand side in Figures 7-11. Solid contour lines represent negative or "core" vorticity, dashed lines are positive or "secondary" vorticity. The contour increment for solid lines not labelled is $-3000 \sec ^{-1} . \omega_{\max }$ is located at some grid point having coordinates $\left(r_{e}, \theta_{c}\right)$. The vortex circulation $\Gamma$ is calculated by first isolating the region of core vorticity in the data field. This is done by referring to the contour plots of vorticity in Figures $7-11$. A path enclosing the region of core vorticity is defined. The outer boundary of the core is taken to be the location where streamwise vorticity is $1 \%$ of $\omega_{\max }$. The circulation is then calculated according to:

$$
\int_{p a t h} \mathrm{~V} \cdot \mathrm{ds}
$$

where $\mathbf{V}$ is the velocity vector in the crossplane, and $s$ refers to the path coordinate. By using closed paths composed of line segments in the $r$ or $\theta$ coordinate directions the circulation is easily determined. Uncertainty estimates for all listed descriptors are given in Table 1. These are derived by combining the uncertainties in measured velocities and probe placement in accordance with the procedure outlined by Moffat. ${ }^{13}$ 


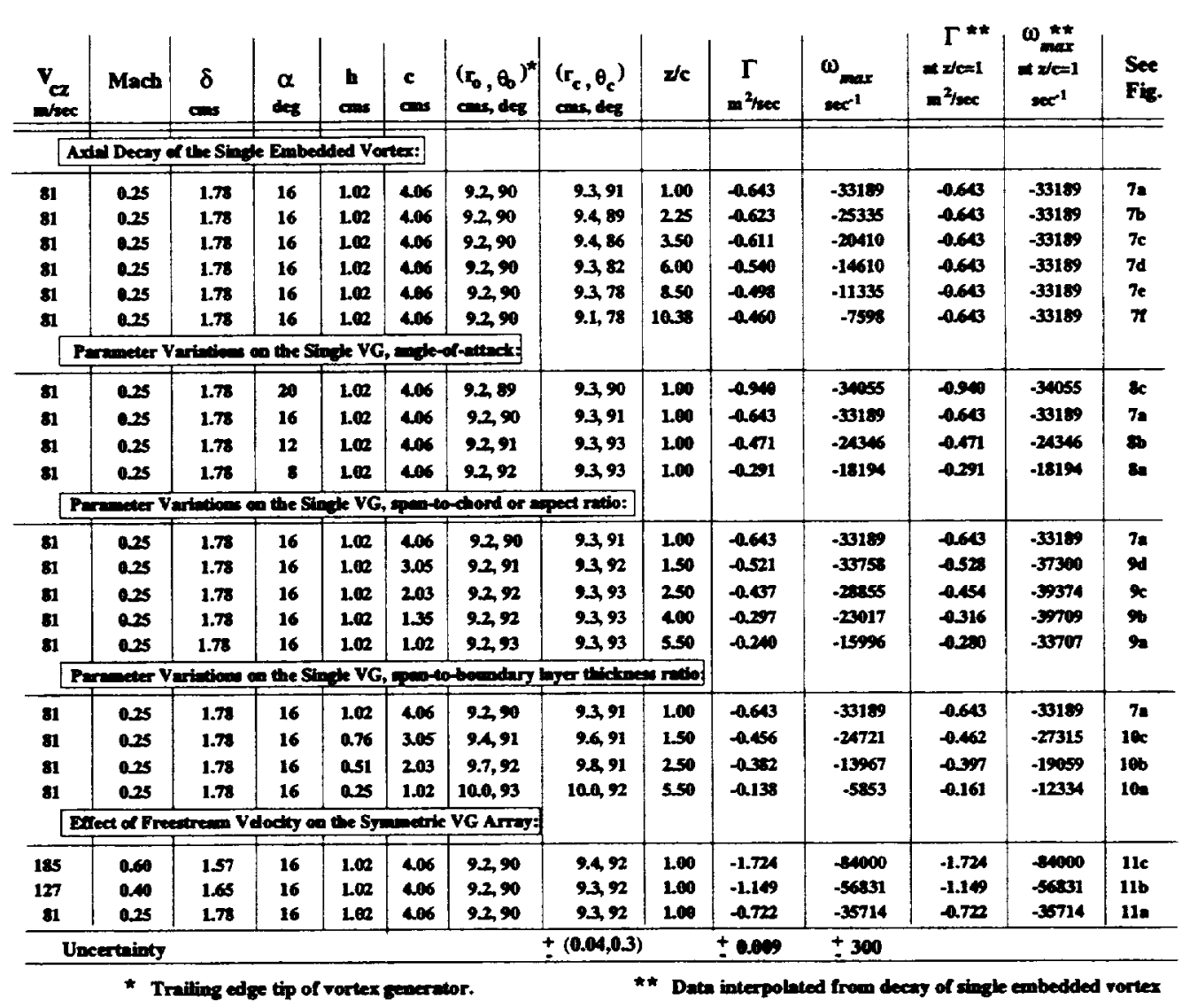

Table 1 - A summary of test conditions and shed vortex descriptor results.

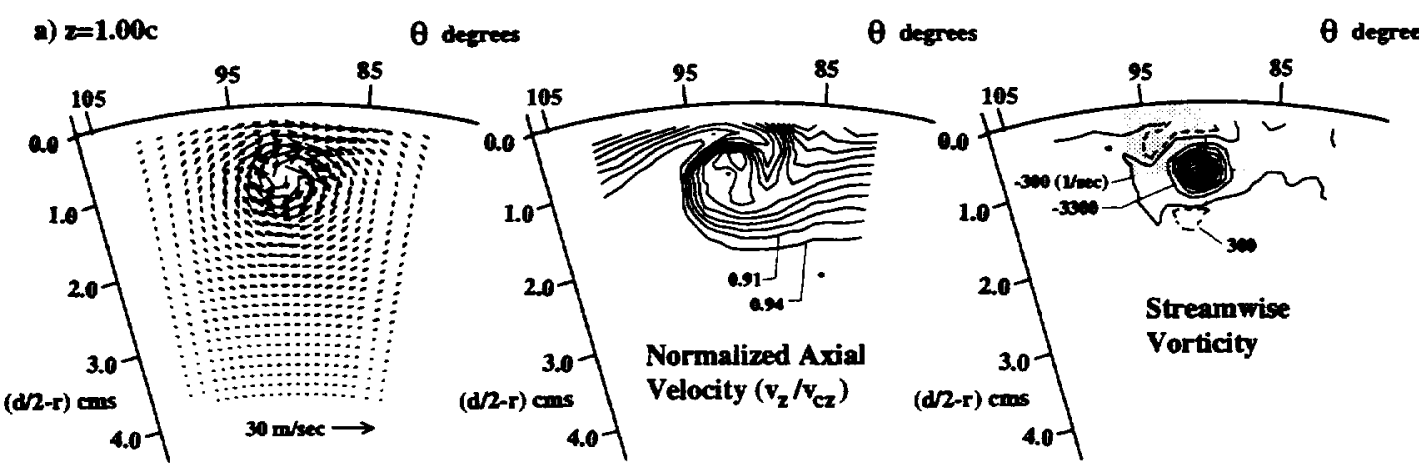

Center of Vortex: $r=9.3 \mathrm{cms}$, theta $=91 \mathrm{deg}$, Circulation $=-0.64 \mathrm{~m} 2 / \mathrm{sec}$, Peak Vorticity $=-33189(1 / \mathrm{sec})$

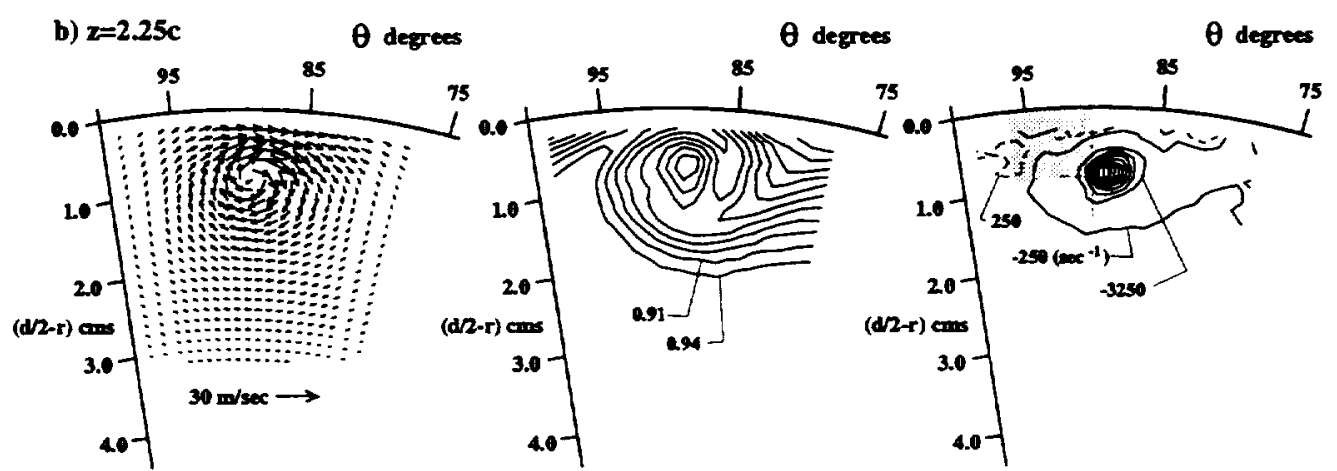

Center of Vortex: $r=9.4 \mathrm{cms}$, theta $=89 \mathrm{deg}$, Circulation $=-0.62 \mathrm{~m} 2 / \mathrm{sec}$, Peak Vorticity $=-25335(1 / \mathrm{sec})$

Figure 7 Velocity and streamwise vorticity results for the axial decay of an isolated embedded vortex. (Continued ....) 


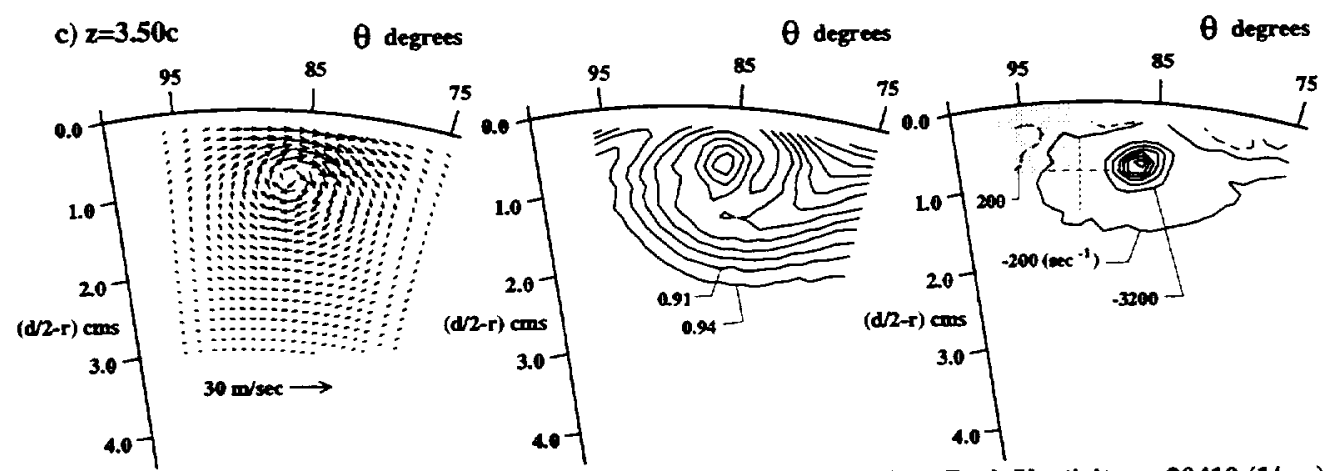

Center of Vortex: $r=9.4 \mathrm{cms}$, theta $=86 \mathrm{deg}$, Circulation $=-0.61 \mathrm{~m} 2 / \mathrm{sec}$, Peak Vorticity $=-20410(1 / \mathrm{sec})$
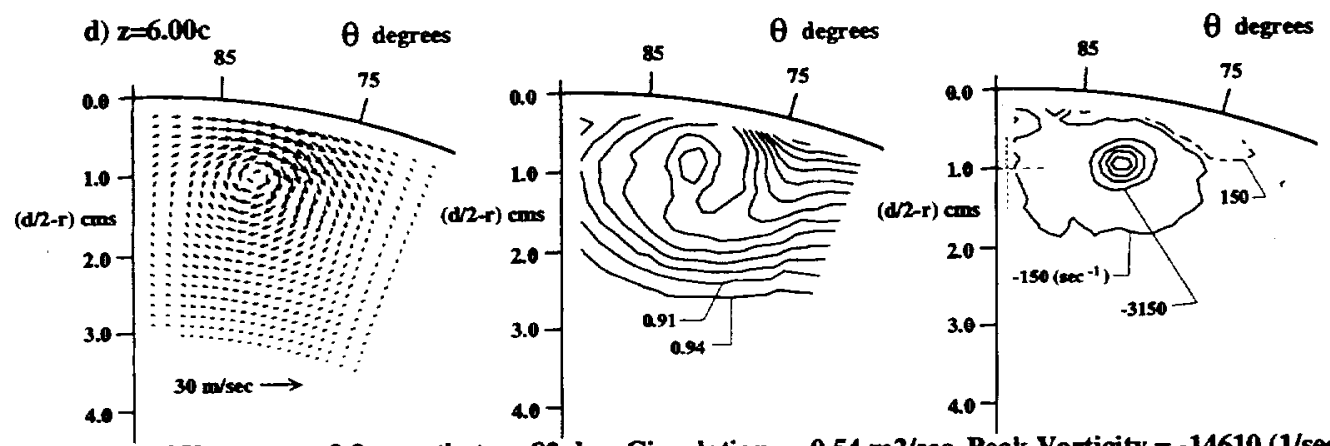

Center of Vortex: $r=9.3 \mathrm{cms}$, theta $=82 \mathrm{deg}$, Circulation $=-0.54 \mathrm{~m} 2 / \mathrm{sec}$, Peak Vorticity $=-14610(1 / \mathrm{sec})$

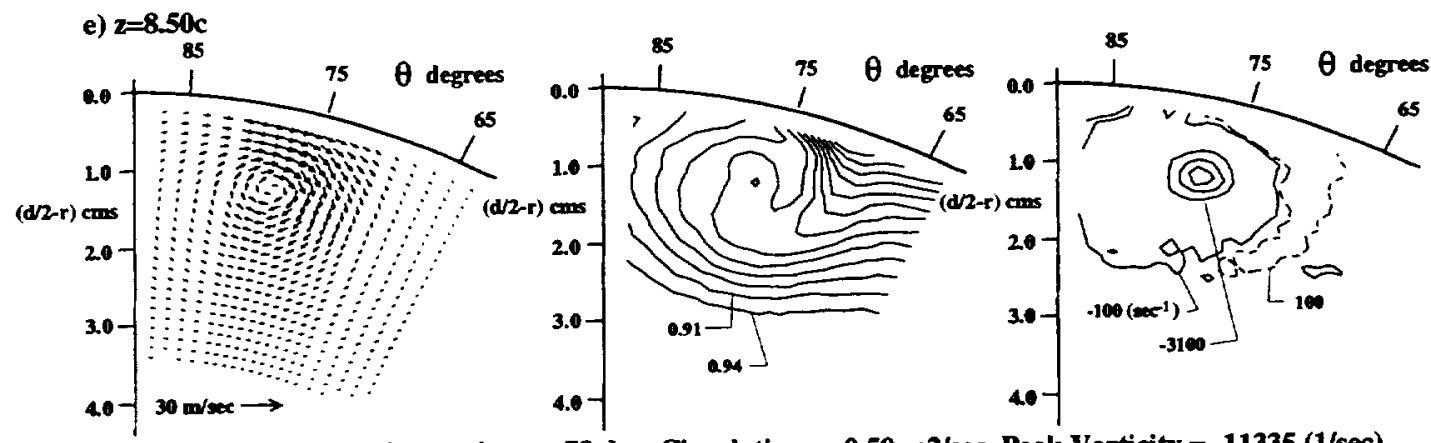

Center of Vortex: $r=9.2 \mathrm{cms}$, theta $=78 \mathrm{deg}$, Circulation $=-0.50 \mathrm{~m} 2 / \mathrm{sec}$, Peak Vorticity $=-11335(1 / \mathrm{sec})$

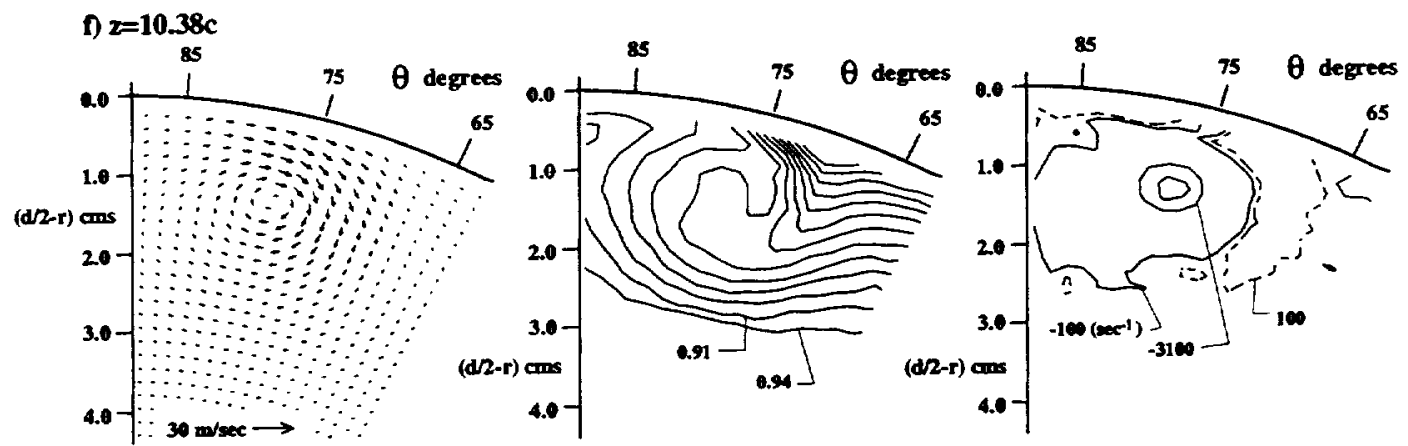

Center of Vortex: $r=9.1 \mathrm{cms}$, theta $=78 \mathrm{deg}$, Circulation $=-0.46 \mathrm{~m} 2 / \mathrm{sec}$, Peak Vorticity $=-7598(1 / \mathrm{sec})$

Figure 7 Velocity and streamwise vorticity results for the axial decay of an isolated embedded vortex. 


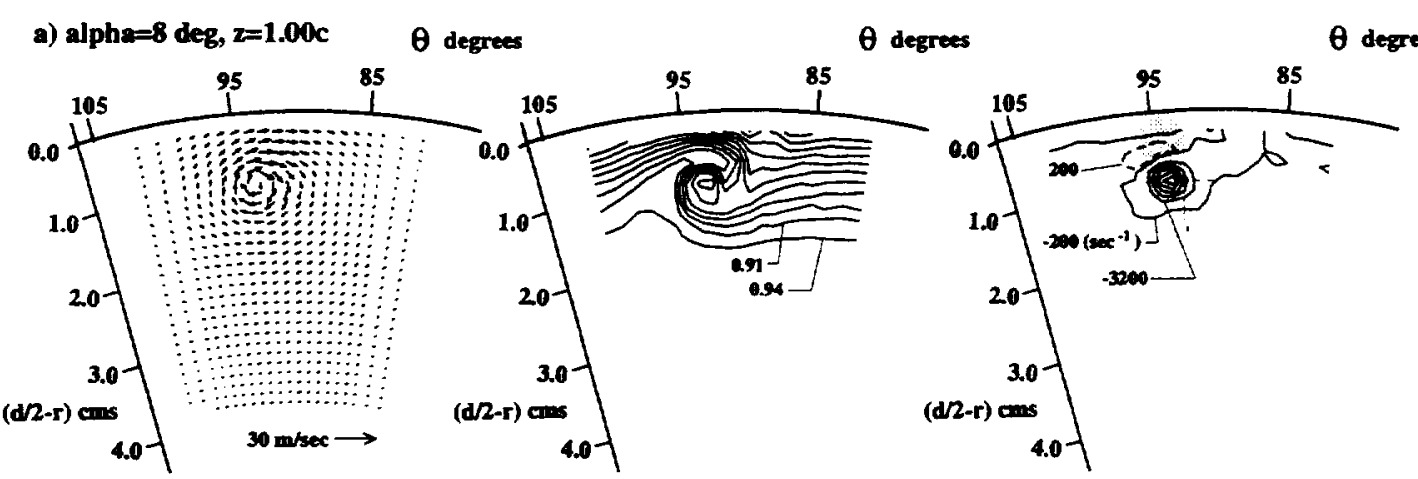

Center of Vortex: $r=9.3 \mathrm{cms}$, theta $=93 \mathrm{deg}$, Circulation $=-0.29 \mathrm{~m} 2 / \mathrm{sec}$, Peak Vorticity $=-18193(1 / \mathrm{sec})$

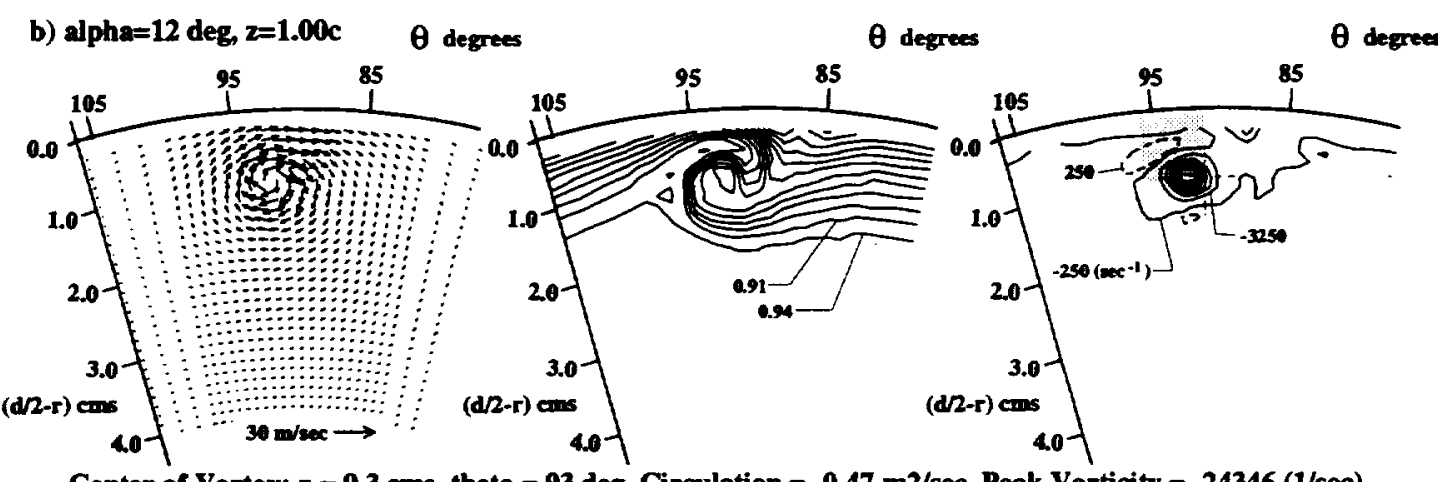

Center of Vortex: $r=9.3 \mathrm{cms}$, theta $=93 \mathrm{deg}$, Circulation $=-0.47 \mathrm{~m} 2 / \mathrm{sec}$, Peak Vorticity $=-24346(1 / \mathrm{sec})$

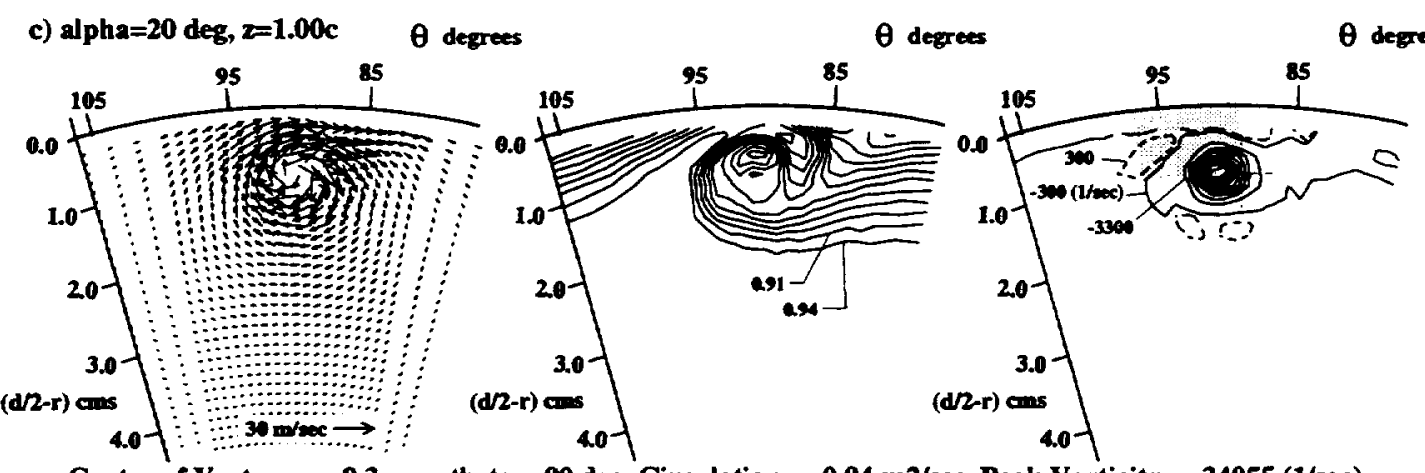

Center of Vortex: $r=9.3 \mathrm{cms}$, theta $=90 \mathrm{deg}$, Circulation $=-0.94 \mathrm{~m} 2 / \mathrm{sec}$, Peak Vorticity $=-34055(1 / \mathrm{sec})$

Figure 8 Velocity and streamwise vorticity results for variation in airfoil angle-of-attack.

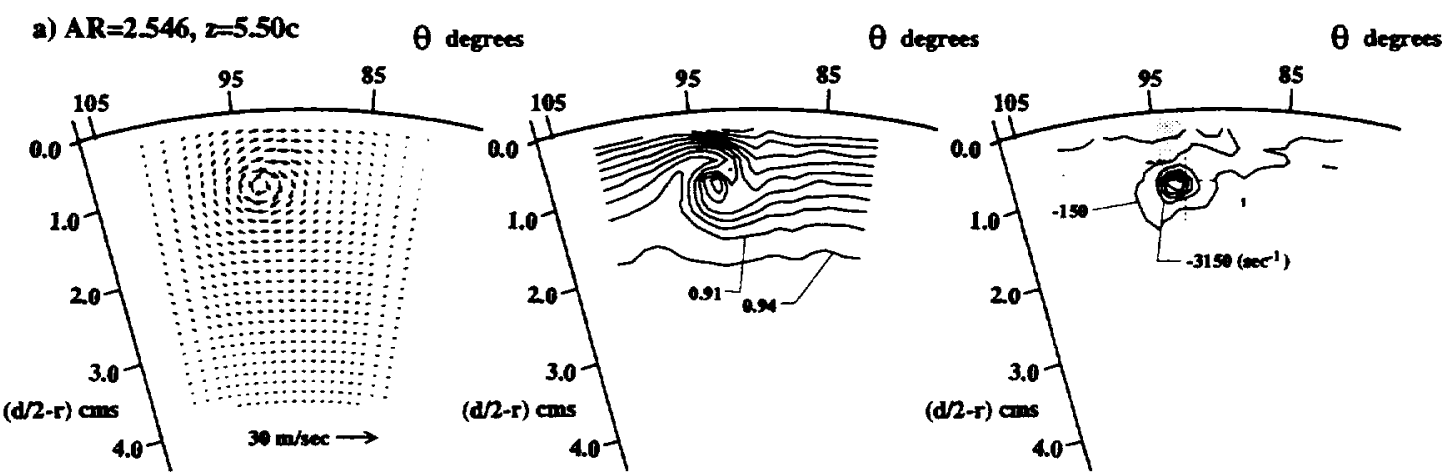

Center of Vortex: $r=9.3 \mathrm{cms}$, theta $=93 \mathrm{deg}$, Circulation $=-0.24 \mathrm{~m} 2 / \mathrm{sec}$, Peak Vorticity $=-15996(1 / \mathrm{sec})$

Figure 9 Velocity and streamwise vorticity results for variation in airfoil aspect ratio. (Continued ....) 


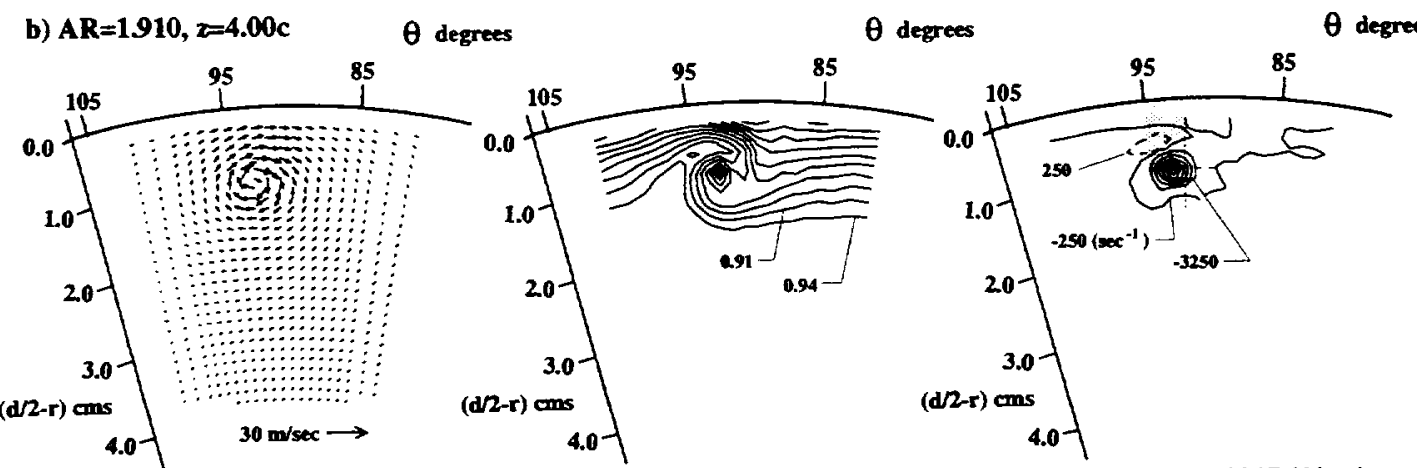

Center of Vortex: $r=9.3 \mathrm{cms}$, theta $=93 \mathrm{deg}$, Circulation $=-0.30 \mathrm{~m} 2 / \mathrm{sec}$, Peak Vorticity $=-23017(1 / \mathrm{sec})$

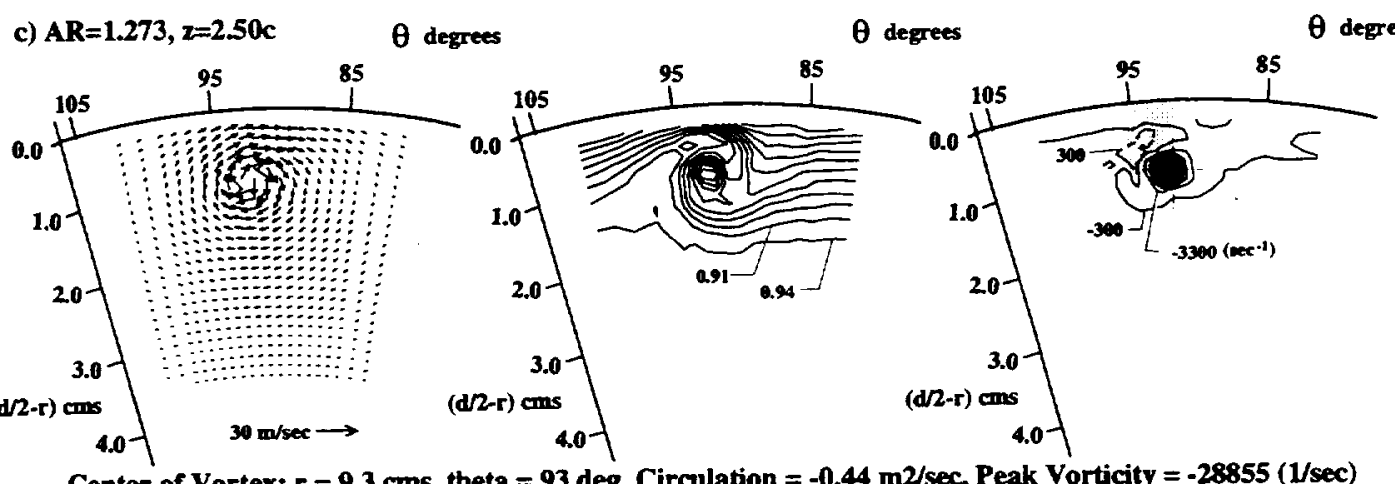

Center of Vortex: $r=9.3 \mathrm{cms}$, theta $=93 \mathrm{deg}$, Circulation $=-0.44 \mathrm{~m} 2 / \mathrm{sec}$, Peak Vorticity $=-28855(1 / \mathrm{sec})$

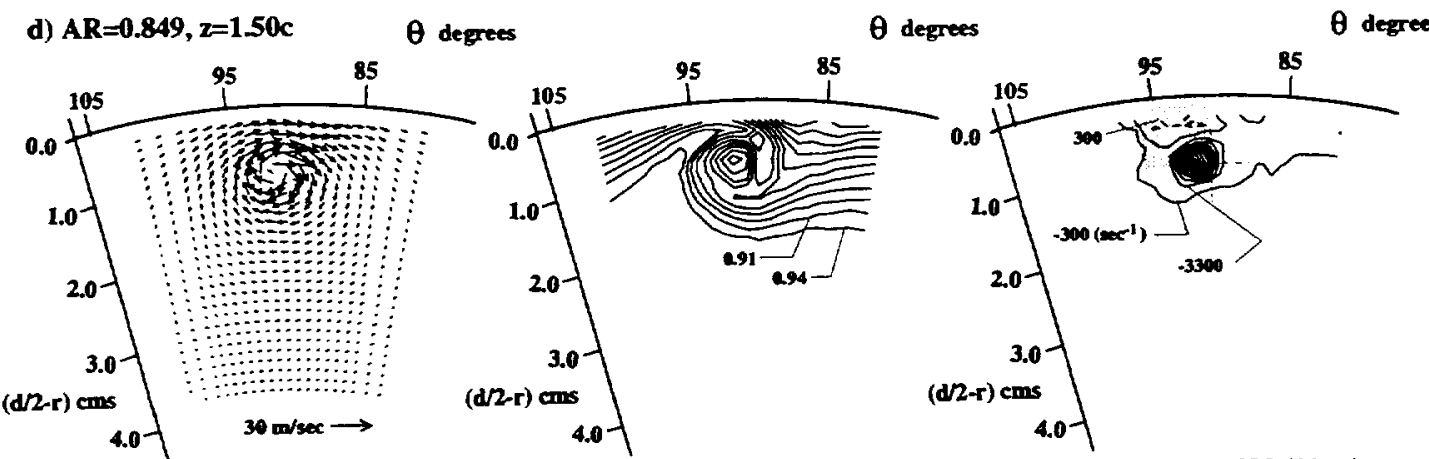

Center of Vortex: $r=9.3 \mathrm{cms}$, theta $=92 \mathrm{deg}$, Circulation $=-0.52 \mathrm{~m} 2 / \mathrm{sec}$, Peak Vorticity $=-33758(1 / \mathrm{sec})$

Figure 9 Velocity and streamwise vorticity results for variation in airfoil aspect ratio.

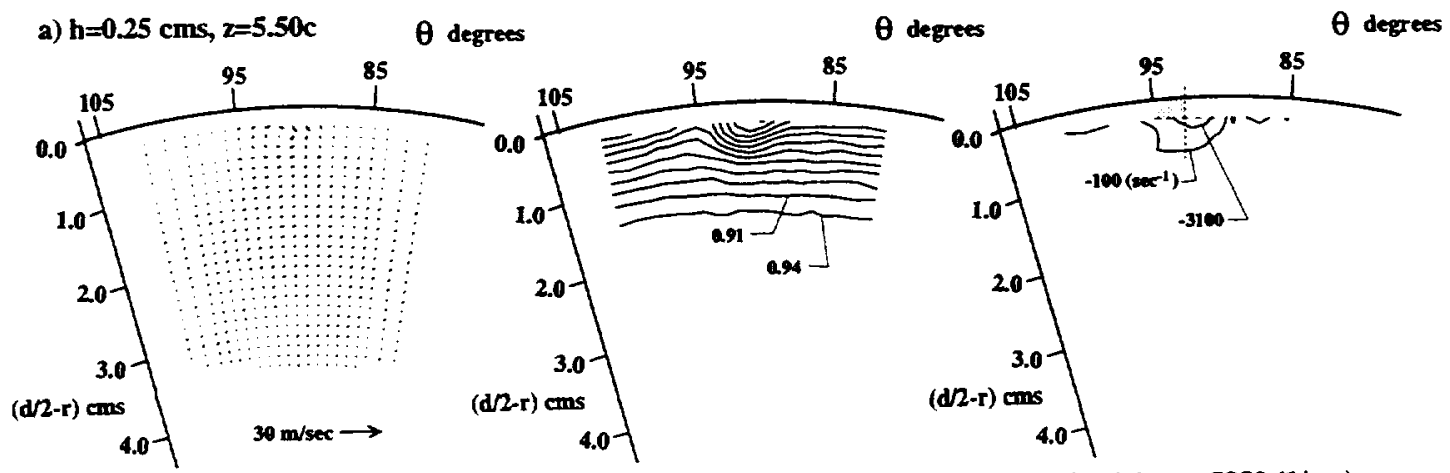

Center of Vortex: $r=10.0 \mathrm{cms}$, theta $=92 \mathrm{deg}$, Circulation $=-0.14 \mathrm{~m} 2 / \mathrm{sec}$, Peak Vorticity $=-5853(1 / \mathrm{sec})$

Figure 10 Velocity and streamwise vorticity results for variation in airfoil span-to-boundary layer thickness ratio. (Continued ....) 


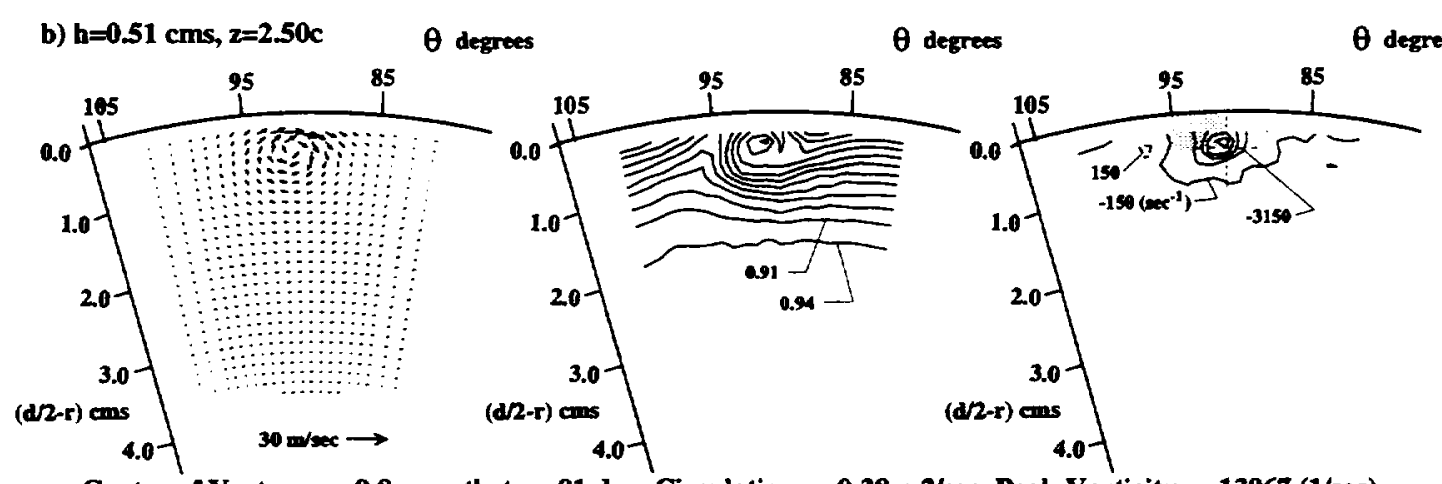

Center of Vortex: $r=9.8 \mathrm{cms}$, theta $=91 \mathrm{deg}$, Circulation $=-0.38 \mathrm{~m} 2 / \mathrm{sec}$, Peak Vorticity $=-13967(1 / \mathrm{sec})$

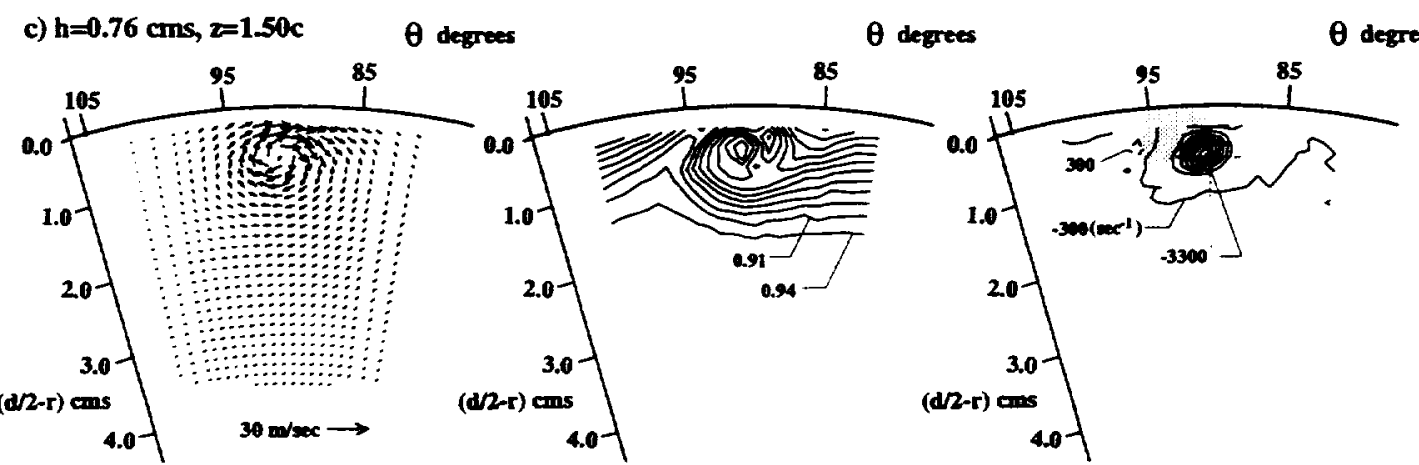

Center of Vortex: $r=9.6 \mathrm{cms}$, theta $=91 \mathrm{deg}$, Circulation $=-0.46 \mathrm{~m} 2 / \mathrm{sec}$, Peak Vorticity $=-24721(1 / \mathrm{sec})$

Figure 10 Velocity and streamwise vorticity results for variation in airfoil span-to-boundary layer thickness ratio.

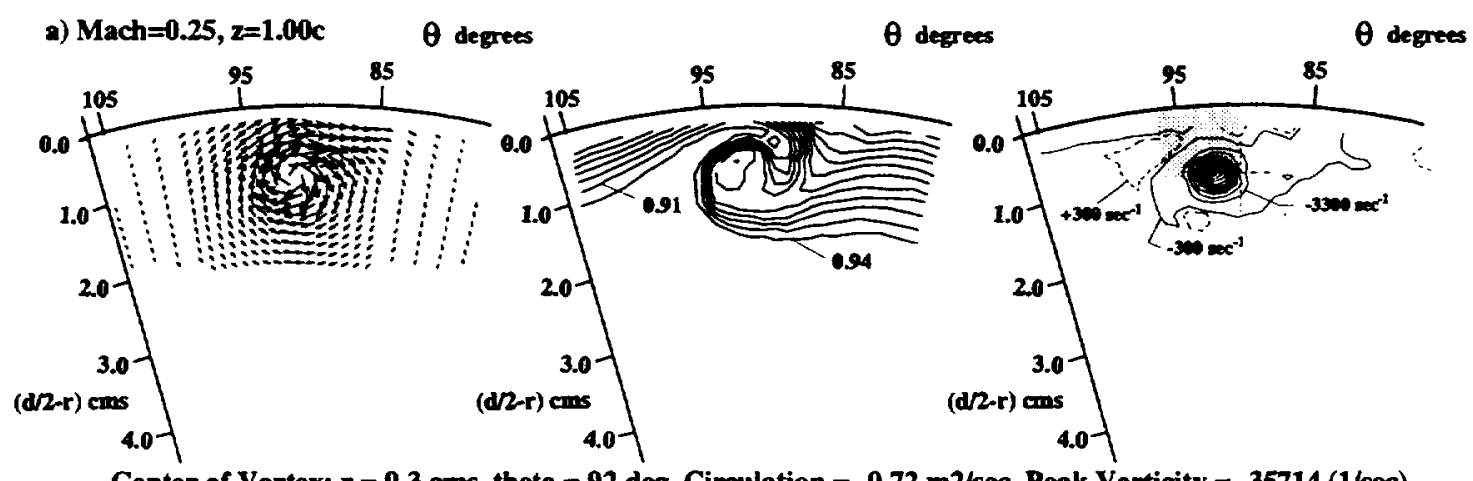

Center of Vortex: $r=9.3 \mathrm{cms}$, theta $=92 \mathrm{deg}$, Circulation $=-0.72 \mathrm{~m} 2 / \mathrm{sec}$, Peak Vorticity $=-35714(1 / \mathrm{sec})$

b) $\mathrm{Mach}=0.40, z=1.00 \mathrm{c}$

$\theta$ degrees

$\theta$ degrees

$\theta$ degrees

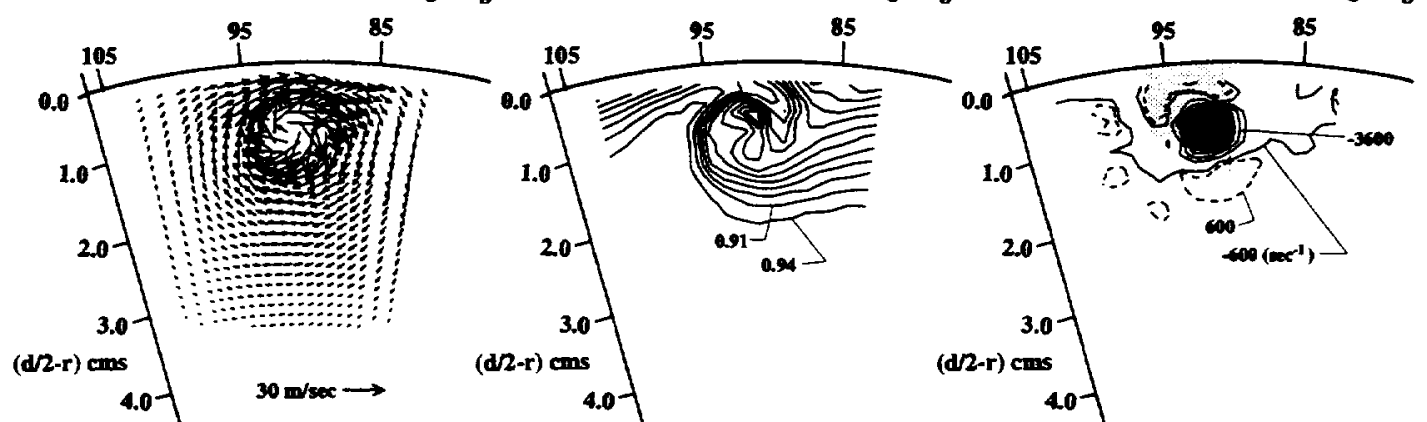

Center of Vortex: $r=9.3 \mathrm{cms}$, theta $=92 \mathrm{deg}$, Circulation $=-1.15 \mathrm{~m} 2 / \mathrm{sec}$, Peak Vorticity $=-56831(1 / \mathrm{sec})$

Figure 11 Velocity and streamwise vorticity results for variation in core Mach number. (Continued ... ) 


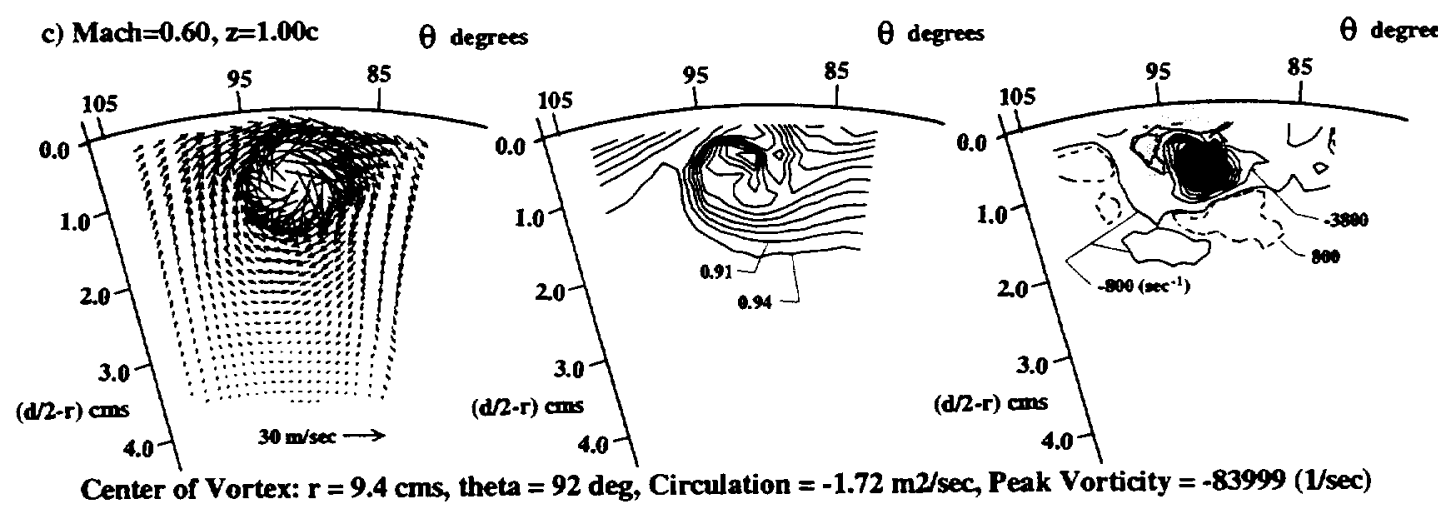

Figure 11 Velocity and streamwise vorticity results for variation in core Mach number.

Contours of primary velocity ratio, $v_{z} / v_{c z}$, are provided in the middle plots of Figures 7-11. $v_{c z}$ is the core velocity of the pipe and is listed in Table 1 . An additional feature of the plots in Figures $7-11$ is the inclusion of the crossplane profile of the vortex generator on the plots of streamwise vorticity (shaded region). The comer marked with the dashed cruciform is the trailing edge tip.

Table 1 is organized into groupings of test cases covering 5 variations in test conditions. The first four groupings examine variation in $z, \alpha$, aspect ratio, and $h / \delta$. Figure $7 \mathrm{a}$, the single embedded vortex at $z=1$ chord and shed from the reference vortex generator, is common to all $\mathbf{4}$ groupings and is thus referred to as the "baseline" test case. This is indicated in Table 1 . The fifth grouping in Table 1, variation in core Mach number, is anchored by results depicted in Figure 11a $(M=0.25)$. The vortex generator array results in Figure 11a are borrowed from Reference 6.

The general nature of the flowfield is illustrated by the three plots in Figure 7a. A concentrated circular vortex structure dominates the flow near the wall, as seen in the transverse velocity and streamwise vorticity plots of Figure $7 \mathrm{a}$. The vortex interaction with the boundary layer is depicted in the contour plot of primary velocity ratio. In the downwash region of the flowfield (to the right of the vortex) the strong secondary flow is convecting a "tongue" of high streamwise momentum fluid under the vortex core, with subsequent boundary layer thinning. Conversely, in the upwash region of the vortex (on the left), the boundary layer fluid is being forced away from the wall, thereby increasing the boundary layer thickness here. A region of primary velocity deficit is also observed to coincide with the center of the vortex core.

\section{Decay of the Single Embedded Vortex}

Figures 7a-f illustrate the development of the single embedded vortex over the axial range: $1.000<z / c<$ 10.375. Figure 12a illustrates the axial decay of circulation, and Figure $12 \mathrm{~b}$ the axial decay of peak vorticity, for both the single embedded vortex and the sym- metric counter-rotating array. The descriptors are nondimensionalized with the values at $z=1$ chord. The uncertainty in the descriptor measurements is indicated by the normal dimension of the plotted symbols (circles and squares). The decay of the single embedded vortex occurs without the influence and interaction of counterrotating neighbors and so the vortex trajectory followed by the isolated vortex is different, leading to a different decay behavior.

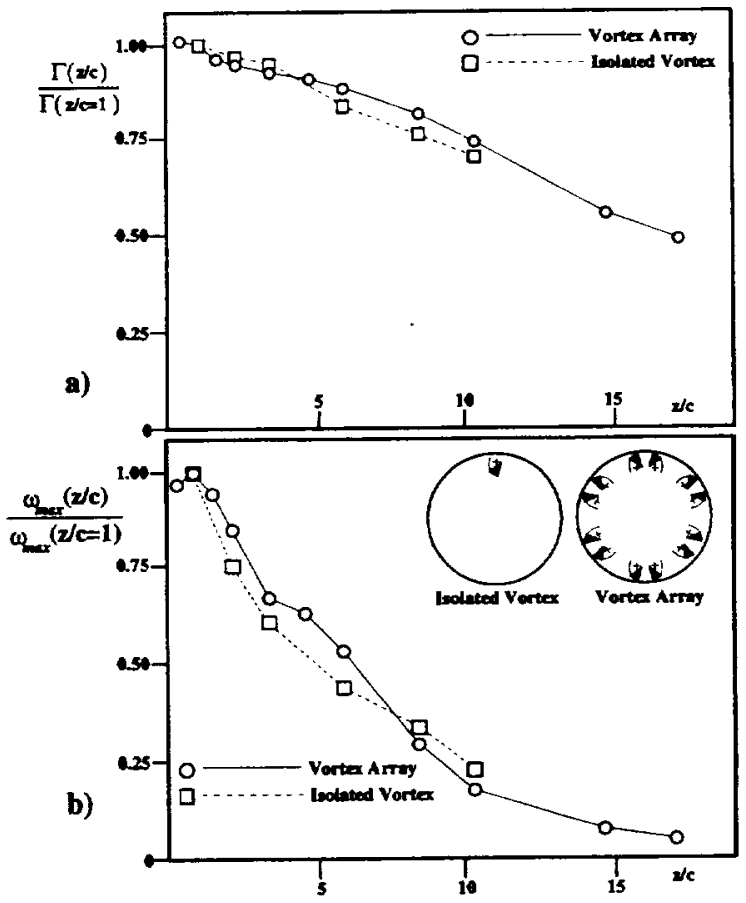

Figure 12 The axial decay of circulation and peak vorticity for both the isolated and array vortex.

Consider, first, the development of circulation. One mechanism of circulation decay is wall friction, which sets up a spanwise component of wall shear stress opposing the rotation of the vortex core. In the array configuration, the vortices are observed to form "upflow" pairs, as depicted in Figures 12b. The upflow pairs tend to convect 
each other away from the wall thereby decreasing circulation losses through wall friction effects. In contrast, the isolated vortex has little tendency to move away from the wall (the small amount of movement observed is due primarily to the growth of the viscous core) and so circulation decay is greater for the isolated vortex over the axial range $1.000<z / c<10.375$.

For vortices in the symmetric array $\omega_{\max }$ reaches its greatest value at $z / c=1.000$. We assume rollup of the shed vortex is complete at this axial location and use $z / c=1.000$ as the reference survey location when studying the influence of vortex generator geometry and flow conditions (as described below) on the vortex descriptors. Downstream of $z / c=1.000$ the decay of $\omega_{\max }$ is very rapid for both the single embedded vortex and the vortices of the symmetric array. This decay decreases rather suddenly at $z / c \approx 3.5$ for array vortices. Note the "hump" in the profile of Figure 12b. Again, this is most likely due to an interaction with the nearest neighbor vortex. Also note that while the decay of $\omega_{\max }$ for the isolated vortex is initially somewhat greater than that of an array vortex, it tapers off downstream of the $z=5 c$ location. Downstream of the $z=8 c$ location the peak vorticity of the isolated vortex is higher than that of the array vortex.

\section{Descriptors Versus Angle-of-Attack}

Figures $7 a$ and Figures $8 a-c$ illustrate the data for variation in angle-of-attack. Figures $13 a-b$ plot the descriptors versus $\alpha$. Circulation increases in proportion to $\alpha$ over the range of $\alpha$ examined $\left(8^{\circ} \leq \alpha \leq 20^{\circ}\right)$. Peak vorticity also increases in proportion to $\alpha$, but only over the range $8^{\circ} \leq \alpha \leq 16^{\circ}$. It is interesting to note that airflow over a two-dimensional NACA0012 airfoil separates at $|\alpha| \approx 16^{\circ} .^{14}$ Perhaps flow separation over the vortex generator is responsible for the flattening of the peak vorticity profile at $\alpha=20^{\circ}$.

Descriptors Versus Aspect Ratio and Span-to-Boundary Layer Thickness Ratio

The aspect ratio of the vortex generator is varied by changing the chordlength of the model. Figure $7 \mathrm{a}$ and Figures 9a-d illustrate the data for variation in chordlength. Aspect ratio, $A R$, is defined as:

$$
\begin{gathered}
A R=4 \times \operatorname{span} /(\pi c)=4 \times(2 h) /(\pi c) \\
=8 h /(\pi c),
\end{gathered}
$$

where span $=2 h$ is used to take into account the wall effect or "image" vortex generator. In acquiring this data, the probe was fixed at one axial station in the instrumentation duct. Thus when the chordlength of the model changed, the axial location of the crossplane data grid, in terms of the model chordlength, changed as well. When studying the effects of model geometry variation it is desirable to have all descriptor data at one axial $(z / c)$
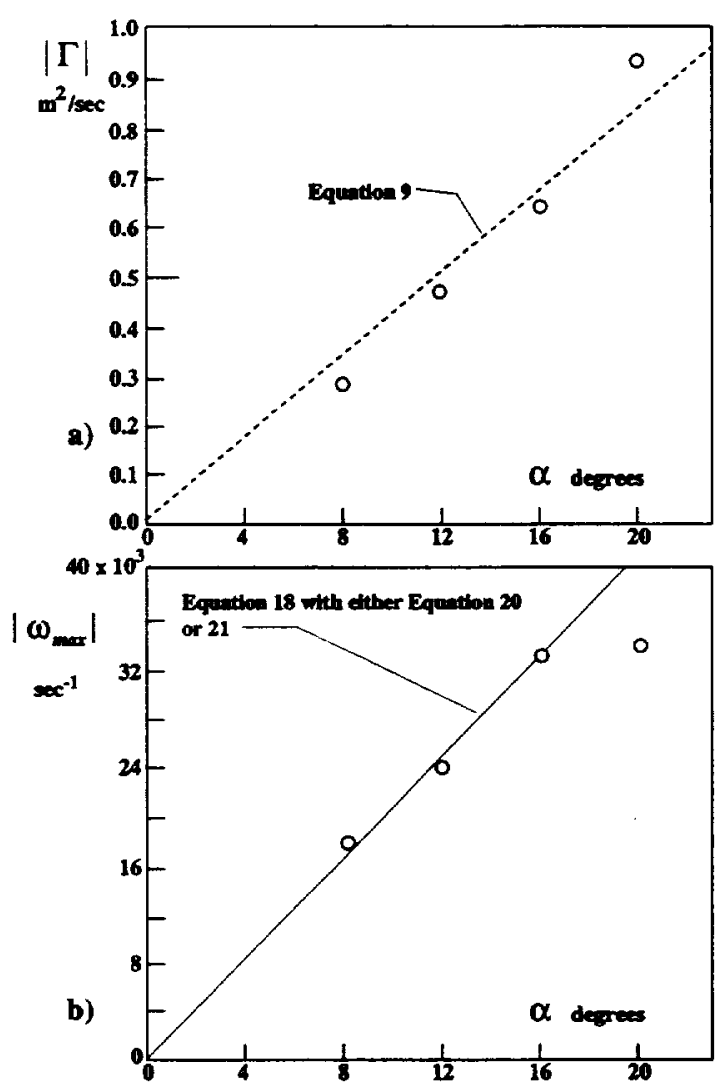

Figure 13 Circulation and peak vorticity plotted against airfoil angle-of-attack.

location. We can estimate the value of the descriptors in this grouping (Figures 9a-d) at the 1 chord location by interpolating the decay behavior of the single embedded vortex discussed earlier. These estimates are done by linear interpolation and are listed in Table 1. Figures 14a-b plot both the raw and interpolated descriptor data versus chordlength (aspect ratio).

The span-to-boundary layer thickness ratio is varied by changing $h$ while holding $A R$ constant. Thus when $h$ is decreased, for example, $c$ is also decreased. Figure $7 a$ and Figures $10 \mathrm{a}-\mathrm{c}$ illustrate the data for variation in $h / \delta$. Again, because the chordlength of the model is changing, the descriptor data has been interpolated to the $z=1 c$ position in Table 1. Both sets of descriptor data have been plotted versus $h / \delta$ in Figures $15 \mathrm{a}-\mathrm{b}$.

\section{Descriptors Versus Core Mach Number}

The variation in core Mach number is conducted for vortices shed in a symmetric counter-rotating arrangement. The arrangement of the parent vortex generator array is illustrated in Figure 6. Figures 11a-c illustrate the data for Mach numbers of $0.25,0.40$, and 0.60 respectively. All data is acquired at the $z=1$ chord location. In Figure 11a the central portion of a data grid 35 degrees in circumferential extent is plotted. This data grid overlaps the domain of the vortex captured there. If all 12 

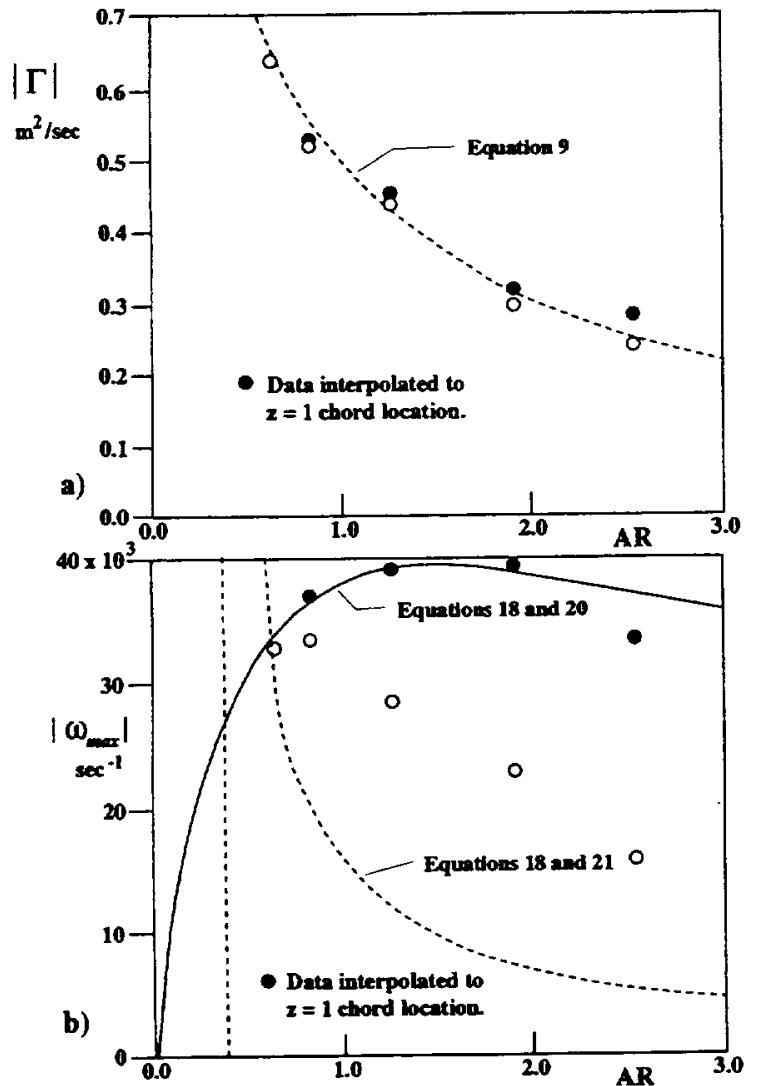

Figure 14 Circulation and peak vorticity plotted against airfoil aspect ratio.

counter-rotating vortices shed from the array of symmetrically placed models illustrated in Figure 6 are assumed to be of equal strength then the term "domain" refers to a $360^{\circ} / 12=30^{\circ}$ circumferential sector of the pipe where the convective influence of one vortex is confined by the action of its neighbors. Measurements conducted over larger segments of the pipe confirm the equality of strength between vortices in the array. In addition to the vortex descriptors, integrated quantities such as vortex angular momentum and transverse kinetic energy may be calculated for the data in Figure 11a. The data grid used in Figures $11 \mathrm{~b}-\mathrm{c}$ cover just over 20 degrees and so do not survey the entire domain of the captured vortex. Figures $16 \mathrm{a}-\mathrm{b}$ plot the variation in descriptors versus core Mach number. Both circulation and peak vorticity are seen to rise in proportion to Mach number.

\section{Analysis and Modelling}

\section{Circulation Descriptor}

The dependence of vortex circulation on vortex generator geometry and impinging flow conditions can be approximated with an expression developed by Prandtl. ${ }^{15}$ For a finite span wing of elliptical planform and symmetric cross-section the circulation developed about the wing

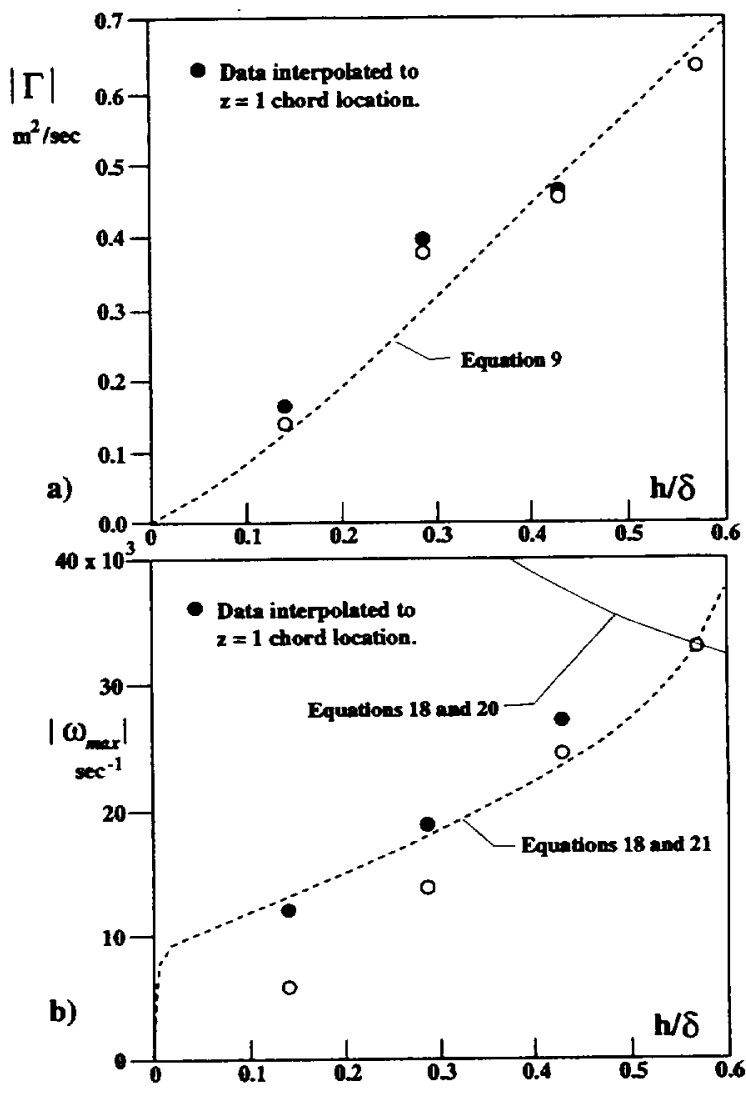

Figure 15 Circulation and peak vorticity plotted against airfoil span-to-boundary layer thickness ratio.

in the plane containing the wing cross-sectional profile is:

$$
\Gamma=\frac{\pi v_{c z} \alpha c}{1+\frac{2}{A R}} .
$$

If we assume that this circulation is turned into the stream (into the duct crossplane) Equation 7 becomes our estimate of the shed vortex circulation. A simple means of accounting for the retarding effect of the boundary layer is to replace $v_{c z}$ with $v$ from the one-seventh power law profile of a turbulent boundary layer:

$$
\frac{v}{v_{c z}}=\left(\frac{h}{\delta}\right)^{\frac{1}{7}}
$$

and, writing Equation 7 in a more general form;

$$
\Gamma=\frac{\kappa_{1} v_{c z} \alpha c}{1+\frac{\kappa_{2}}{A R}} \cdot\left(\frac{h}{\delta}\right)^{\frac{1}{7}} .
$$

The constants $\kappa_{1}$ and $\kappa_{2}$ are determined from the circulation data using a least squares procedure:

$$
\kappa_{1}=1.55, \kappa_{2}=0.637 \text {. }
$$

Equation 9 is plotted against the data in Figures 13a16a. Flow conditions and vortex generator geometry are taken from values listed in Table 1 and used in Equation 9 in a manner consistent with the test conditions. So, for example, in Figure 13a, $v_{c z}$ is held to $81 \mathrm{~m} / \mathrm{sec}$, $c=4.064 \mathrm{cms}, h=1.016 \mathrm{cms}, \delta=1.778 \mathrm{cms}$, and $A R=0.637$. We see that Equation 9 correlates the data well in every case. 

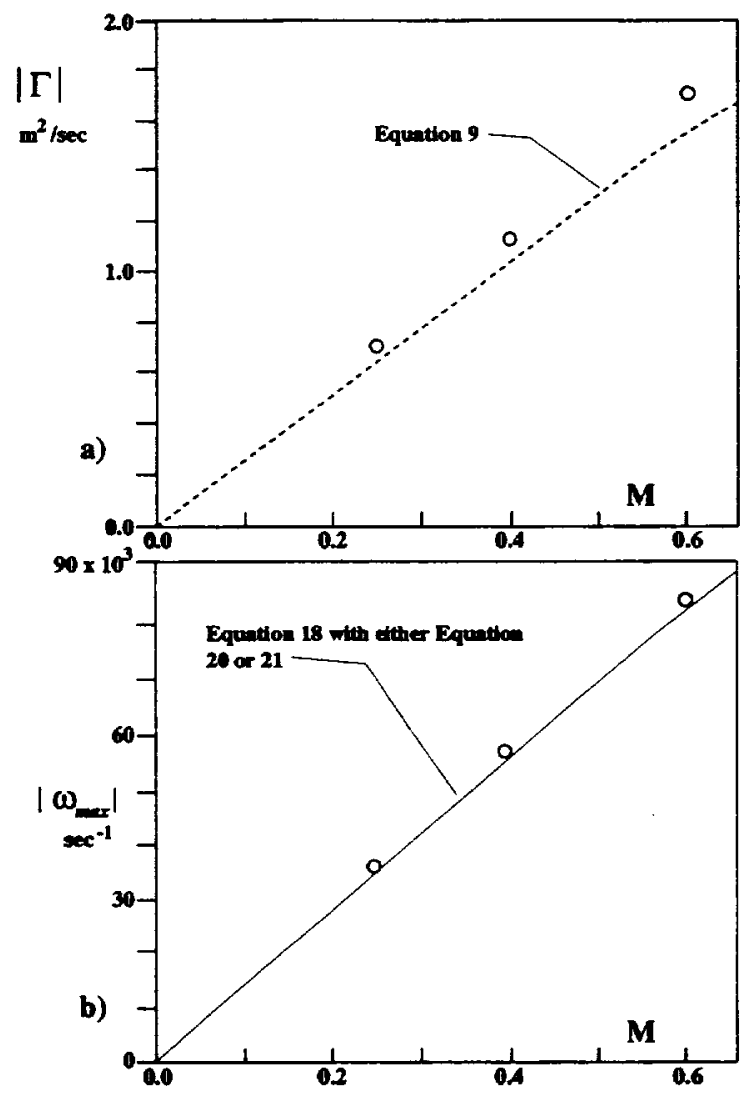

Figure 16 Circulation and peak vorticity plotted against core Mach number.

\section{Peak Vorticity Descriptor}

Suppose that the mysterious behavior of peak vorticity illustrated in Figures $13 \mathrm{~b}-16 \mathrm{~b}$ is connected to a vortex image or wall effect. A correlation for the peak vorticity descriptor at the $z=1$ chord location is now developed below following these further assumptions:

1. The characteristic moment in the $(r, \theta)$ crossplane can be equated to the rate of angular momentum production by the shed vortex. This moment is taken to be $L \cdot t$, where $L$ is the lift force acting on the vortex generator, and $t$ is the airfoil thickness, $t=0.012 c$. The angular momentum, $H_{v}$, of the shed vortex about its center of rotation is determined from:

$$
\frac{H_{v}}{\rho \cdot d z}=\int_{\text {domain }} \vec{r}_{\mathrm{c}} \times \vec{v} d A,
$$

where $\vec{r}_{c}$ is the radial position measured from the center of the vortex, $\vec{v}$ is the transverse velocity vector, and "domain" refers to the entire crossplane of the vortex generator duct.

2. A vortex model accurately represents the secondary velocity structure of the vortex (see Figure 2) at the $z=1$ chord location.

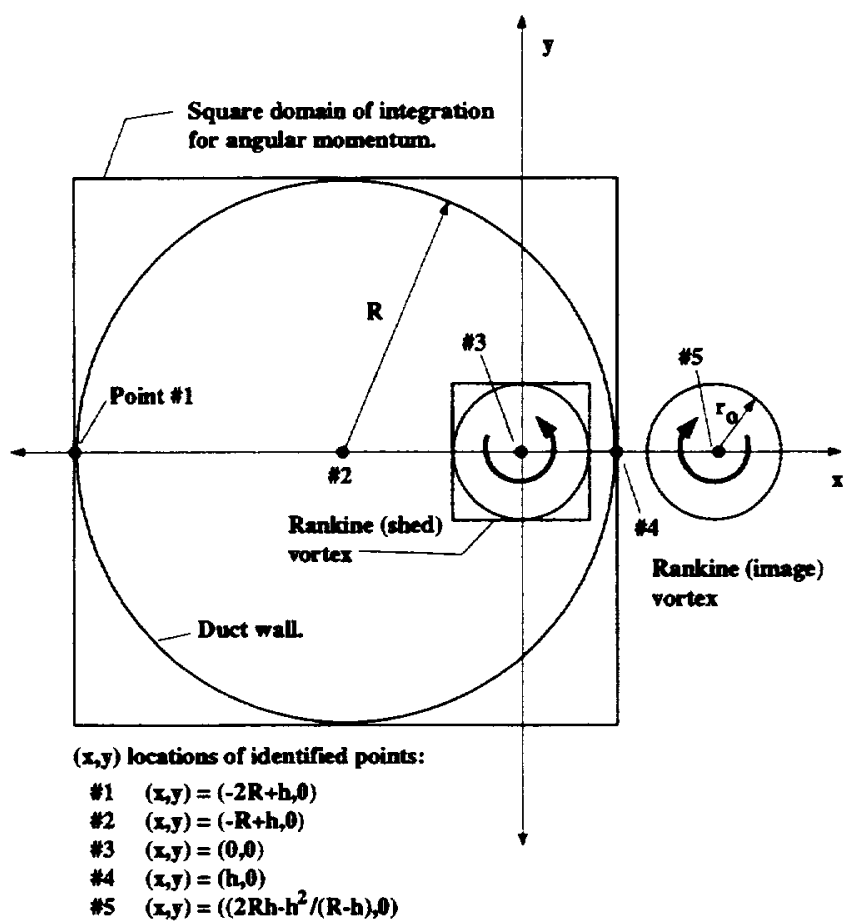

Fig. 17 A model of an isolated vortex in a circular duct using a superpostion of two Rankine vortices.

Several additional simplifications are necessary to integrate Equation 11 and obtain a closed form solution similar to Equation 9. These are shown in Figure 17. Figure 17 illustrates the vortex model and domain of integration used to calculate the angular momentum. The vortex model consists of two superimposed Rankine vortices. The Rankine model is used here so that the integration in Equation 11 may be evaluated in closed form. The vortex on the left is the modelled (shed) vortex, the vortex on the right is an image vortex positioned to represent the effect of the duct wall on the flowfield of the shed vortex. The Rankine model is a "patchwork" vortex model with the following definition:

$$
\begin{gathered}
v_{\theta}=\frac{\Gamma r}{2 \pi r_{0}^{2}}, \quad\left(r \leq r_{0}\right),(\text { viscous core) } \\
v_{\theta}=\frac{\Gamma}{2 \pi r}, \quad\left(r>r_{0}\right),(\text { inviscid outer field). }
\end{gathered}
$$

The radius of the viscous core $r_{o}$, the vortex circulation, and peak vorticity are related as follows:

$$
r_{o}^{2}=\frac{\Gamma}{\pi \omega_{\max }} .
$$

The domain of integration for Equation 11 are the rectangular regions enclosing the circular duct and vortex core. With these approximations the angular momentum of the vortex model is:

$$
\frac{H_{v}}{\rho \cdot d z} \approx \frac{\Gamma}{2 \pi}\left[A(R, h)-\frac{4 \Gamma}{\pi \omega_{\max }}\right],
$$


where:

$$
\begin{gathered}
A(R, h)=\left(\frac{2 R h-h^{2}}{R-h}\right)\left[\frac{2 R(h-2 R)}{R-h} \arctan \left(\frac{R-h}{h-2 R}\right)\right. \\
+\frac{2 R h}{R-h} \arctan \left(\frac{h-R}{h}\right)+ \\
\left.\quad R \ln \left(\frac{2 h^{2}-6 R h+5 R^{2}}{2 h^{2}-2 R h+R^{2}}\right)\right],
\end{gathered}
$$

subject to the restrictions: $\left(r_{0} \leq h\right),(h<R)$. Proceeding to the moment equation:

$$
\begin{gathered}
\sum M_{v}=\frac{\delta H_{v}}{\delta t} \approx \frac{v_{c z} H_{v}}{d z}=L \cdot t=C_{L} \frac{1}{2} \rho v_{c z}^{2}(2 h) c t \\
\text { or } C_{L} v_{c z} h c t=\frac{\Gamma}{2 \pi}\left[A(R, h)-\frac{4 \Gamma}{\pi \omega_{\max }}\right] .
\end{gathered}
$$

An expression for $C_{L}$ is borrowed from inviscid (finite wing) airfoil theory:

$$
C_{L}=\frac{2 \kappa_{1} \alpha}{\left(1+\frac{\kappa_{2}}{A R}\right)}
$$

where " $\pi$ " has been replaced with " $\kappa_{1}$ " and "2" with " $\kappa_{2}$ " as in Equation 9. Substitute Equations 6, 9, and 17 into Equation 16, replace $v_{c z}$ with $v$ in Equation 8, and substitute $t=0.012 c$ to obtain:

$$
\begin{gathered}
\left|\omega_{\max }\right| \approx \frac{\beta_{o} v_{c z} \alpha c h}{(4 h+c)\left(A(R, h)-\beta_{1} h c\right)} \cdot\left(\frac{h}{\delta}\right)^{\frac{1}{7}}, \\
\beta_{o}=7.89, \beta_{1}=1.51 .
\end{gathered}
$$

Compare this expression to Equation 9 written in terms of $h$ and $c$ :

$$
\Gamma \approx \frac{6.20 v_{c z} \alpha c h}{(4 h+c)} \cdot\left(\frac{h}{\delta}\right)^{\frac{1}{7}} .
$$

Due to the approximate nature of the analysis only the functional form displayed by Equation 18 is of interest and so the particular values of the constants $\beta_{0}$ and $\beta_{1}$ are discarded. To explore this functional form further two new values of $\beta_{1}$ are chosen, and $\beta_{o}$ is determined (for each value of $\beta_{1}$ ) by fitting Equation 18 to the baseline data point (Figure 7a). The two sets of constants are:

$$
\beta_{0}(1)=16422, \quad \beta_{1}(1)=-110,
$$

and:

$$
\beta_{\circ}(2)=920, \quad \beta_{1}(2)=10 .
$$

Equation 18, with both sets of constants, is plotted against the data in Figures $13 \mathrm{~b}-16 \mathrm{~b}$ in a manner similar to the procedure followed for Equation 9 and Figures 13a-16a. We note first the proportionality between $\omega_{\max }, \alpha$, and $v_{c z}$ displayed by Equation 18. In Figure 13 a we see this correlation represents the data well over the range $8^{\circ} \leq \alpha \leq 16^{\circ}$. This is also true for variation in $v_{c z}$ or $M$ displayed by Figure $16 \mathrm{~b}$. For variation in $A R$ and $h / \delta$ the two sets of constants in Equation 18 produce correlations that are distinctly different. In Figure $14 \mathrm{~b}$ and $15 \mathrm{~b}$ the solid lines are Equations 18 and 20 , the dashed lines are Equations 18 and 21 . Equations 18 and 20 mimic the interpolated data well in Figure 14b but do not represent the behavior of $\omega_{\max }$ versus $h / \delta$ in Figure 15b. Equations 18 and 21 do a better job of representing $\omega_{\max }$ versus $h / \delta$ (particularly the interpolated data) in Figure $15 \mathrm{~b}$ but largely underestimate $\omega_{\max }$ in Figure 14b. Different values of $\beta_{o}$ and $\beta_{1}$ in Equation 18 do not alter this pattern of correlation.

\section{Summary}

An experimental study is conducted to examine the crossplane flow structure of longitudinal vortices shed from symmetric airfoil vortex generators. The primary goal of this study is to establish the dependence of vortex generator geometry and impinging flow conditions on the shed vortex descriptors of circulation and peak vorticity at an axial station 1 chord downstream of the vortex generator trailing edge. The airfoils have a NACA 0012 cross-sectional profile and are mounted either in isolation or in a symmetric counter-rotating array on the inside surface of a straight pipe. The impinging flow conditions are subsonic, atmospheric air with a turbulent boundary layer thickness to pipe radius ratio $\delta / R \approx 0.17$. Measurements of mean three-component velocities in the crossplane are used to derive the shed vortex circulation and peak vorticity. Vortex generator angle-of-attack is varied over the range: $8^{\circ} \leq \alpha \leq 20^{\circ}$, aspect ratio over the range: $0.64 \leq A R \leq 2.55$, span-to-boundary layer thickness ratio over the range: $0.14 \leq h / \delta \leq 0.57$, and impinging Mach number over the range: $0.25 \leq M \leq 0.60$.

Vortex circulation is observed to increase in proportion to $\alpha, M$, and $h / \delta$. When these parameters are beld constant, and airfoil aspect ratio is varied, circulation is seen to fall off in monotonic fashion with increasing aspect ratio.

The behavior of vortex peak vorticity is similar to that of circulation, with two exceptions:

1. Peak vorticity increases in proportion to $\alpha$ up to $\alpha \approx 16^{\circ}$ only and flattens off thereafter.

2. Peak vorticity rises in monotonic fashion with airfoil aspect ratio reaching a maximum value at $A R \approx 2.0$ and falling off thereafter.

The influence of $\alpha, M, h / \delta$, and $A R$ on the shed vortex circulation can be well approximated by an empirical relation derived from Prandtl's formula (Equation 9).

The influence of $\alpha$ and $M$ on the shed vortex peak vorticity is represented by a correlation developed from a moment-balance in the spanwise plane of the shed vortex. The influence of $A R$ and $h / \delta$ cannot be represented simultaneously with this correlation and is likely an indication that something more than an image or wall effect 
is responsible for the behavior of peak vorticity versus $A R$ and $h / \delta$.

\section{Acknowledgements}

This project was supported with funding from NASA Lewis and the National Research Council. The authors would like to acknowledge the skillful support provided by many. Appreciated were the mechanical skills of William Darby and Bob Ehrbar (from NASA's Test Installation Division) and the systems engineering support of Robert Gronski (NYMA). Charles Wasserbauer (also of NYMA) provided engineering and operational support. Design engineering service was provided by Arthur Sprungle of NASA Lewis. Dr. Warren R. Hingst provided valuable insight into the test section design and Bernie Anderson aided this effort with his computations of vortex generator flowfields and his enthusiasm for the subject. Jeffry Foster (lowa State graduate assistant) belped in acquiring the data. Finally, a special thank you to Mr. T. Nugent who kept our collective noses to the grindstone throughout the long course of this project.

\section{References}

${ }^{1}$ Reichert, B. A. and Wendt, B. J., "Improving Diffusing S-Duct Performance by Secondary Flow Control," AIAA Paper 94-0365, Jan. 1994.

${ }^{2}$ Foster, J., Okiishi, T. H., Wendt, B. J., and Reichert, B. A., "Study of Compressible Flow Through a Rectangular-to-Semiannular Transition Duct," NASA CR 4660, Apr. 1995.

${ }^{3}$ Anderson, B. H. and Farokhi, S., "A Study of Three Dimensional Turbulent Boundary Layer Separation and Vortex Flow Control Using the Reduced Navier-Stokes Equations," Turbulent Shear Flow Symposium tech. rep., 1991.

${ }^{4}$ Anderson, B. H. and Gibb, J., "Application of Computational Fluid Dynamics to the Study of Vortex Flow
Control for the Management of Inlet Distortion," AIAA Paper 92-3177, July 1992.

${ }^{5}$ Cho, S. Y. and Greber, I., Three Dimensional Compressible Turbulent Flow Computations for a Diffusing S-Duct WithWithout Vortex Generators, Ph.D. Dissertation, Case Western Reserve University, Cleveland, OH, Nov. 1992.

${ }^{6}$ Wendt, B. J., Reichert, B. A., and Foster, J. D., "The Decay of Longitudinal Vortices Shed from Airfoil Vortex Generators," AIAA Paper 95-1797, June 1995.

${ }^{7}$ Westphal, R. V., Pauley, W. R., and Eaton, J. K., "Interaction Between a Vortex and a Turbulent Boundary Layer-Part 1: Mean Flow Evolution and Turbulence Properties," NASA TM 88361, Jan. 1987.

${ }^{8}$ Wendt, B. J. and Hingst, W. R., "Flow Structure in the Wake of a Wishbone Vortex Generator," AlAA Joumal, Vol. 32, Nov. 1994, pp. 2234-2240.

${ }^{9}$ Pauley, W. R. and Eaton, J. K., “The Fluid Dynamics and Heat Transfer Effects of Streamwise Vortices Embedded in a Turbulent Boundary Layer," Stanford University Tech. Rep. MD-51, Stanford, CA, Aug. 1988.

${ }^{10}$ Wendt, B. J., Greber, I., and Hingst, W. R., "The Structure and Development of Streamwise Vortex Arrays Embedded in a Turbulent Boundary Layer," AIAA Paper 92-0551, Jan. 1992.

${ }^{11}$ Porro, A. R., Hingst, W. R., Wasserbauer, C. A., and Andrews, T. B., "The NASA Lewis Research Center Internal Fluid Mechanics Facility," NASA TM 105187, Sept. 1991.

${ }^{12}$ Zilliac, G. G., "Modelling, Calibration, and Error Analysis of Seven-Hole Probes," Experiments in Fluids, Vol. 14, 1993, pp. 104-120.

${ }^{13}$ Moffat, R. J., "Contributions to the Theory of SingleSample Uncertainty Analysis," Transactions of the ASME, Vol. 104, June 1982, pp. 250-258.

${ }^{14}$ Abbott, I. H. and Doenhoff, A. E. V., Theory of Wing Sections, 2nd ed, Dover, New York, 1959.

${ }^{15}$ Prandtl, L., "Applications of Modern Hydrodynamics to Aeronautics," NACA Report 116, 1921. 


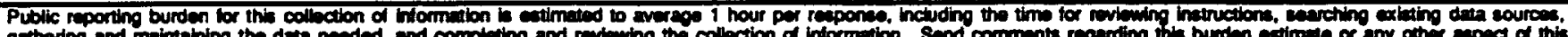

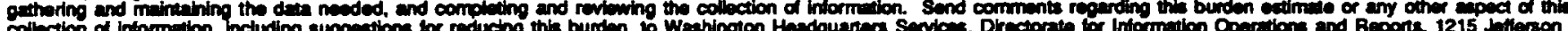

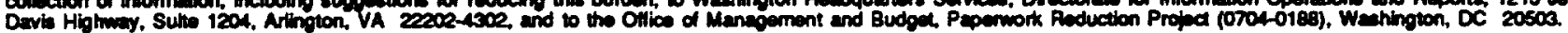

\begin{tabular}{|l|l|l}
\hline 1. AGENCY USE ONLY (Leave blank) & $\begin{array}{r}\text { 2 FEPOFT DATE } \\
\text { June } 1996\end{array}$ & $\begin{array}{r}\text { 3. REPOFT TYPE AND DATES COVERED } \\
\text { Final Contractor Report }\end{array}$ \\
\hline
\end{tabular}

4. TITE AND SUBTIRE

5. FUNDING NUMBERS

The Modelling of Symmetric Airfoil Vortex Generators

WU-505-62-52

6. AUTHOP(S)

C-NAS3-27377

B.J. Wendt and B.A. Reichert

7. PERFORING ORGANZATION NAME(S) AND ADDRESS(ES)

Modern Technologies Corporation

Cleveland Office

7530 Lucerne Drive

Islander Two, Suite 206

Middleburg Heights, Ohio 44130

9. SPONSOFINGMONTORING AGENCY MAME(S) AND ADDRESS(ES) AGENCY REPORT NUMBER

National Aeronautics and Space Administration

Lewis Research Center

Cleveland, Ohio 44135-3191

NASA CR-198501

AIAA-96-0807

\section{SUPPLENENTARY NOTES}

Prepared for the 34th Aerospace Sciences Meeting and Exhibit, Reno, Nevada, January 15-18, 1996. B.J. Wendt, Modern Technologies Corporation, Middleburg Heights, Ohio 44130 and B.A. Reichert, Kansas State University, Manhattan, Kansas 66506. Project Manager, John M. Abbot, NASA Lewis Research Center, Internal Fluid Mechanics Division, organization code 2660 , (216) 433-3607.

12a. DISTRIBUTKONAVALABILTY STATEMENT

12b. DISTPABUTION CODE

Unclassified - Unlimited

Subject Category 02

This publication is available from the NASA Center for AeroSpace Information, (301) 621-0390.

13. ABSTRACT (Noximum 200 words)

An experimental study is conducted to determine the dependence of vortex generator geometry and impinging flow conditions on shed vortex circulation and crossplane peak vorticity for one type of vortex generator. The vortex generator is a symmetric airfoil having a NACA 0012 cross-sectional profile. The geometry and flow parameters varied include angle-of-attack $\alpha$, chordlength $c$, span $h$, and Mach number $M$. The vortex generators are mounted either in isolation or in a symmetric counter-rotating array configuration on the inside surface of a straight pipe. The turbulent boundary layer thickness to pipe radius ratio is $\delta R=0.17$. Circulation and peak vorticity data are derived from crossplane velocity measurements conducted at or about 1 chord downstream of the vortex generator trailing edge. Shed vortex circulation is observed to be proportional to $M, \alpha$, and $h / \delta$. With these parameters held constant, circulation is observed to fall off in monotonic fashion with increasing airfoil aspect ratio $A R$. Shed vortex peak vorticity is also observed to be proportional to $M, \alpha$, and $h / \delta$. Unlike circulation, however, peak vorticity is observed to increase with increasing aspect ratio, reaching a peak value at $A R \approx 2.0$ before falling off.

\section{SUBJECT TERMS}

15. MUMBER OF PAGES

Vortices; Vortex generators; Three-dimensional boundary layer

17. SECUATY CLASSIFICATION OF REPOAT Unclassified

18. SECURTY CLASSIFICATION OF THS PACE

Unclassified
19. SECUATY CLASSIFICATION OF ABSTRACT

Unclassified 


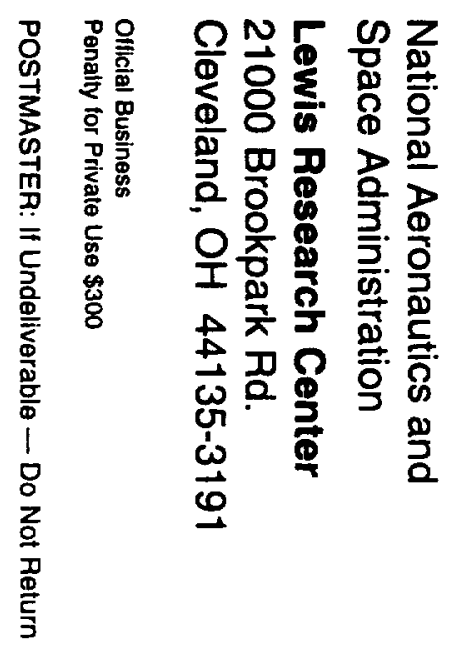

